\title{
MicroRNAs as potential biomarkers in malignant pleural mesothelioma
}

This article was published in the following Dove Press journal:

Current Biomarker Findings

23 December 2015

Number of times this article has been viewed

\section{Eric Santoni-Rugiu \\ Morten Andersen \\ Morten Grauslund \\ Laboratory of Molecular Pathology, Department of Pathology, \\ Rigshospitalet, Copenhagen University Hospital, Copenhagen, Denmark}

Correspondence: Eric Santoni-Rugiu Laboratory of Molecular Pathology, Department of Pathology, Section 5442, Rigshospitalet, Copenhagen University Hospital, Blegdamsvej 9, 2100

Copenhagen, Denmark

Tel +45 35455476

Fax +4535455414

Email eric.santoni-rugiu.02@regionh.dk
Abstract: Malignant pleural mesothelioma (MPM), a highly lethal cancer strictly related to asbestos exposure, is usually characterized by delayed diagnosis, resistance to current therapies, and dismal prognosis. MPM is difficult to distinguish histologically from nonmalignant reactive mesothelial proliferations (RMPs) as there are no clinically validated immunohistochemical markers yet and the main diagnostic criterion remains deep invasion into the pleura and underlying fat tissue, which is often not appreciable in small pleural biopsies. In this regard, microRNAs (miRNAs), given their size and stability, are particularly attractive biomarkers in formalin-fixed paraffin-embedded tissue specimens for routine pathology. Moreover, circulating miRNAs appear to be promising biomarkers for early detection and monitoring of patients with MPM. Here, we review the studies mostly performed by miRNA arrays and reverse transcription-quantitative polymerase chain reaction in formalin-fixed paraffin-embedded or frozen tissue samples, MPM cell lines, and blood/plasma/serum samples that have highlighted the potential of miRNAs as biomarkers in MPM. Certain studies have pointed to the ability of miRNAs to distinguish the different histological MPM subtypes or separate MPM from lung adenocarcinoma, and other investigations have revealed that miRNAs can aid in differentiating MPM from RMP or have prognostic value in predicting the patient outcome. Mechanistic aspects of the involvement of miRNAs in mesothelioma genesis and possible use of miRNAs as future therapeutic targets in MPM are also emphasized. Finally, limitations of the data currently obtained due to the drawbacks of reverse transcription-quantitative polymerase chain reaction, heterogeneity of MPM tissue samples, and differences in methodological platforms as well as in types of specimens utilized in different studies are discussed. Because of these inherent weaknesses of collected data, further studies assessing the expression and distribution of miRNAs by in situ hybridization in combination with the codetection of their respective targets by immunohistochemistry and further validation of miRNAs' targets in vitro are warranted to fully reveal the potential clinical utility of miRNAs in MPM.

Keywords: malignant pleural mesothelioma, miRNAs, cancer biomarkers, diagnosis, prognosis, future therapy

\section{Introduction}

Malignant pleural mesothelioma (MPM), a rare, aggressive cancer originating from the mesothelial cells lining the pleura, is in most cases related to long-term exposure to asbestos or asbestos-like fibers. Histologically, MPM is classified as epithelioid malignant pleural mesothelioma (EMPM), sarcomatoid malignant pleural mesothelioma (SMPM), and biphasic malignant pleural mesothelioma (BMPM) subtypes representing $70 \%, 10 \%$, and $20 \%$ of cases, respectively. ${ }^{1} \mathrm{MPM}$ is most often diagnosed at an advanced stage, where standard treatment is palliative platinum-based chemotherapy. 
Consequently, the prognosis remains dismal with a median survival of 12 months after diagnosis. ${ }^{1}$ For $\sim 30 \%$ of patients judged to be operable at diagnosis, specialized centers offer cytoreductive surgical procedures, such as extrapleural pneumonectomy or pleurectomy/decortication, often combined in a trimodal protocol with neoadjuvant chemotherapy and adjuvant radiotherapy. ${ }^{2}$ According to MPM histology, which remains the most significant prognostic factor, operable patients with EMPM, BMPM, and SMPM have a median survival of 19,13 , and 8 months, respectively. ${ }^{1}$ MPM typically responds poorly to chemoradiotherapy, and proposed biomarkers for identifying the patients who would most benefit from these treatments need more clinical validation. ${ }^{3}$ Recent efforts focused on identifying efficacious molecular targeted therapy and immunotherapy hold promise for the implementation of more personalized protocols in the near future. ${ }^{4}$ MPM can be a difficult diagnosis for pathologists. The main diagnostic criterion remains deep invasion into the pleura and underlying fat tissue, which is often not discernable histologically in small pleural biopsies. Effective immunohistochemistry (IHC) algorithms can most often be successfully applied to distinguish MPM from pleural spreading of other cancer types, such as lung cancer. ${ }^{1}$ However, it can be quite challenging to distinguish EMPM from nonmalignant reactive mesothelial hyperplasia or SMPM from organizing pleuritis ${ }^{1}$ as proposed IHC biomarkers for malignant mesothelial cells have not shown enough specificity and/or sensitivity or require further validation. ${ }^{5,6}$ Thus, the identification of new biomarkers that may aid in the problematic diagnosis and/or treatment of MPM is urgently needed. By summarizing the several overlapping general definitions existing in the literature for simplicity, biomarkers can be considered as objectively and reproducibly measurable characteristics of biological (physiological or pathological) processes.

In this respect, a class of noncoding RNAs, known as microRNAs (miRNAs), has shown great potential in other types of cancers as diagnostic, prognostic, and predictive biomarkers (biomarkers that provide information regarding an individual patient's diagnosis, prognosis regardless of treatment, and likelihood to respond to a specific therapy, respectively). ${ }^{7,8}$ miRNAs are short RNA strands of 18-24 nucleotides that regulate the gene expression by base pairing to complementary sequences primarily within the 3 '-untranslated regions (3'-UTR) of target mRNAs, thereby resulting in mRNA cleavage or translational repression. ${ }^{9}$ The latest version of the miRBase (http://www.mirbase. org/) miRNA-registry contains 2,588 entries of mature human miRNAs, which are estimated to target 3 '-UTRs in $>60 \%$ of human mRNAs. ${ }^{9,10}$ Each miRNA can modulate the expression of hundreds of target mRNAs, and when its expression is deregulated, it can act as tumor suppressor or oncogene ("oncomiR"), depending on the nature of its targets. Indeed, miRNAs are involved in the regulation of multiple fundamental cellular activities, including proliferation, apoptosis, differentiation, senescence, invasion, motility, stress responses, and metabolism, which are all closely linked to cancer initiation, progression, and treatment response. ${ }^{11}$ miRNAs are particularly attractive as biomarkers in tissue samples processed for routine pathology as their short nucleotide sequences remain stable and can be quantified in formalin-fixed paraffin-embedded (FFPE) material by polymerase chain reaction (PCR) and in situ hybridization (ISH) techniques. ${ }^{12}$ Similarly, they are detectable in the blood, in which they circulate mostly as exosomal vesicles, thereby representing promising potential noninvasive biomarkers for cancer diagnostics and follow-up. ${ }^{13}$ Here, we review the recent discoveries regarding miRNA expression and function in MPM, with special emphasis on the potential clinical use as future biomarkers (Figure 1). Table 1 summarizes experimental details and significant miRNA findings in the studies exploring the biomarker potential of miRNAs in MPM. Table 2 summarizes the studies disclosing predicted and verified target genes of deregulated miRNAs in MPM.

\section{miRNAs with deregulated expression and diagnostic potential in MPM}

Several studies, mostly based on initial screening by miRNA microarrays and validation by reverse transcriptionquantitative polymerase chain reaction (RT-qPCR), have explored the expression of miRNAs in MPM. ${ }^{14-20}$

The first study demonstrating deregulated miRNA expression in MPM was that by Guled et al, ${ }^{14}$ who screened the expression of 723 human miRNAs in total RNA isolated from snap-frozen MPM samples (eleven EMPM, five BMPM, one SMPM) and in commercially available total RNA from normal human pericardium. The MPM tissues displayed overexpression of 12 miRNAs and underexpression of nine miRNAs (Table 1). The study then portrayed the exclusive expression of seven miRNAs in EMPM (miR-135b, -181a$\left.2^{*},-499-5 p,-517 b,-519 d,-615-5 p,-624\right)$, five in BMPM (miR-218-2*, -346, -377*, -485-5p, -525-3p), and three in the only SMPM analyzed (miR-301b, -433, -543). Utilizing target-prediction algorithms, the tumor suppressor genes (TSGs), CDKN2A and NF2, which are commonly inactivated 


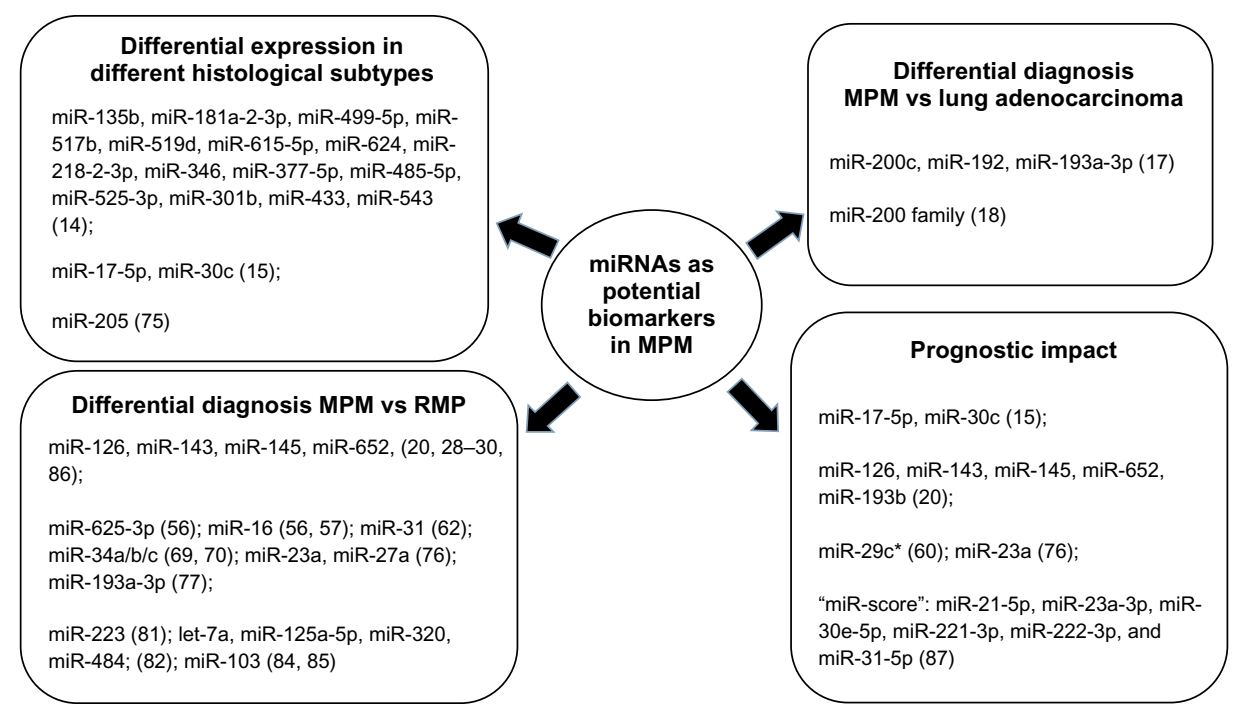

Figure I MicroRNAs (miRNAs) with potential as clinical biomarkers in malignant pleural mesothelioma (MPM).

Notes: miRNAs that can distinguish the histological subtypes of MPM, differentiate MPM from lung adenocarcinoma, aid in the differential diagnosis of MPM vs nonmalignant reactive mesothelial proliferation (RMP), or that have displayed prognostic significance for MPM patients are shown. Corresponding references for reported findings on the different miRNAs are shown in brackets (references).

in MPM, ${ }^{1}$ and $R B 1$ were identified as putative targets of the overexpressed miR-30b*, $-32 *,-483-3 p,-584$, and $-885-3 p .^{14}$ Similarly, the underexpressed miR-9, -7-1*, and -203 were predicted to target the oncogenes $H G F, P D G F A, E G F$, and JUN (Table 2). ${ }^{14}$

A microarray-based miRNA profiling in vitro by Busacca et al displayed upregulation of ten miRNAs and downregulation of 19 miRNAs in two commercially available MPM cell lines as compared to immortalized human mesothelial cells (HMC). ${ }^{15}$ Validation by RT-qPCR showed consistent overexpression of $\mathrm{miR}-17-5 \mathrm{p}$ and $\mathrm{miR}-30 \mathrm{c}$ and underexpression of miR-221 and miR-222 in the MPM cell lines. The ten most differentially expressed miRNAs were tested by RT-qPCR in a collection of 24 MPM samples representing each histological subtype of MPM, aiming at uncovering miR signatures that could differentiate these subtypes. However, it is somehow disputable that the expression of the miRNAs was reported relative to a reference sample obtained by pooling an equal amount of RNA from each specimen. ${ }^{15}$ The expression of seven of the tested miRNAs was found to be significantly associated with the histological subtypes, and for SMPM, the cases with lower expression of miR-17-5p and miR-30c (below the median) were associated with better prognosis. $^{15}$

By comparing the expression of 470 human and 63 viral miRNAs in primary HMC and five commercially available MPM cell lines, Balatti et al identified eight underexpressed miRNAs and 15 overexpressed miRNAs in the MPM cells. ${ }^{16}$ Among the overexpressed miRNAs were seven members of the miR-17-92 cluster (including miR-17-5p, thus, reproducing the upregulation of this miRNA observed by Busacca et al in different MPM cell lines) $)^{15}$ and its paralogs miR-106a-363 and miR-106b-25. A few of the dysregulated miRNAs were validated. It was also demonstrated that in MPM cells, as in other cancer cell lines, the miR-17-92 cluster targets the tumor suppressor $C D K N 1 A$ gene, which encodes the cyclindependent kinase inhibitor $\mathrm{p} 21 .^{16}$

Other comprehensive studies in vivo focused instead on tissue-specific miR signatures that could discriminate MPM from lung adenocarcinoma. ${ }^{17,18} \mathrm{~A}$ diagnostic test based on differentially expressed miRNAs, which allegedly discerns MPM from peripheral adenocarcinomas of the lung with high sensitivity and specificity, is currently being marketed by Rosetta Genomics Ltd (Rehovot, Israel) under the commercial name miRview ${ }^{\mathrm{TM}}$ meso. The test was established after comparing the expression of miRNAs in FFPE specimens of 33 MPMs and 210 adenocarcinomas of different organs and detecting the underexpression of seven miRNAs and overexpression of four miRNAs in MPMs. ${ }^{17}$ Three of these miRNAs (miR-192, -193a-3p, -200c) were further validated in an expanded set of tissue samples, leading to a diagnostic protocol that by combining the overexpression of miR-200c and -192 in lung adenocarcinomas and overexpression of miR-193a-3p in MPM could distinguish these two malignancies with $100 \%$ sensitivity and $94 \%$ specificity. ${ }^{17}$ This study emphasized the downregulation of the miR-200 family components (miR-141, -200a/b/c, -429) in MPM vs lung adenocarcinoma. ${ }^{17}$ Gee et al confirmed the potential value 







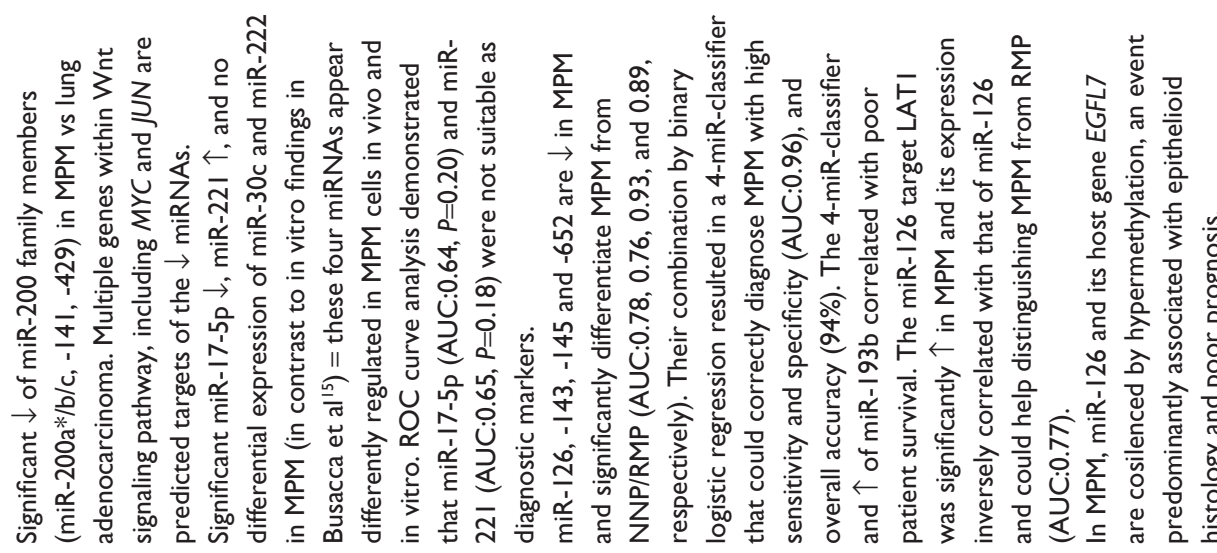

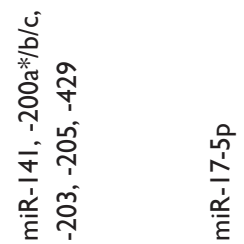

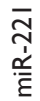

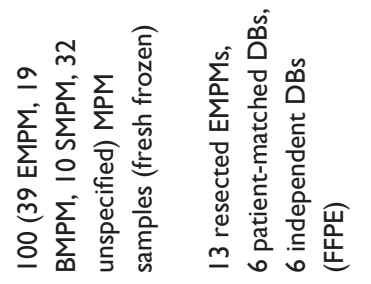

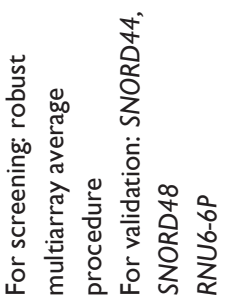

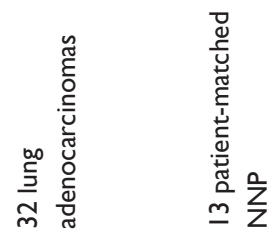

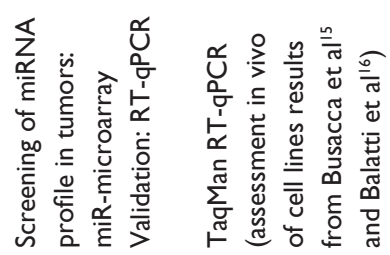

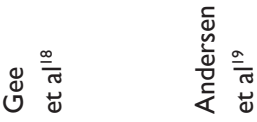

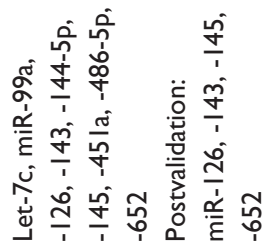

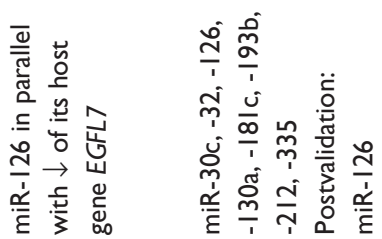

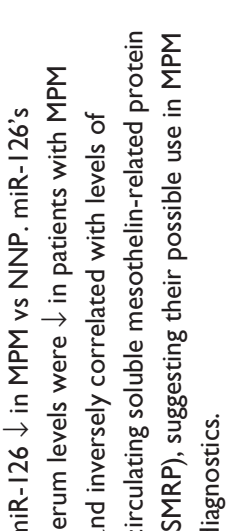

है

ำ ำ

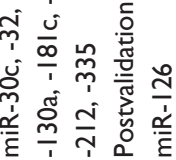

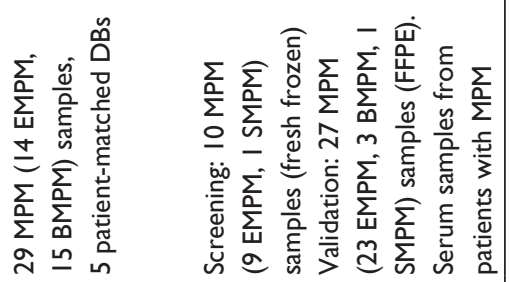

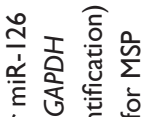

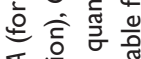

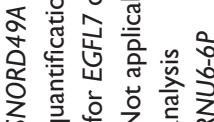

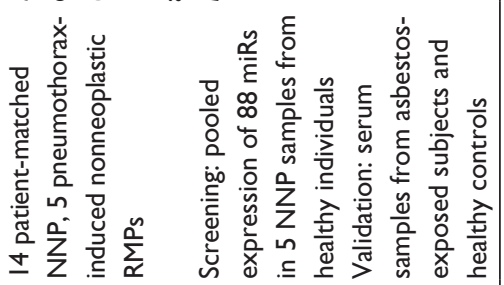

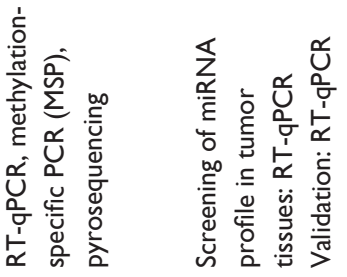

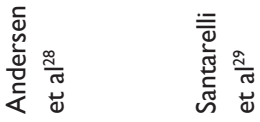




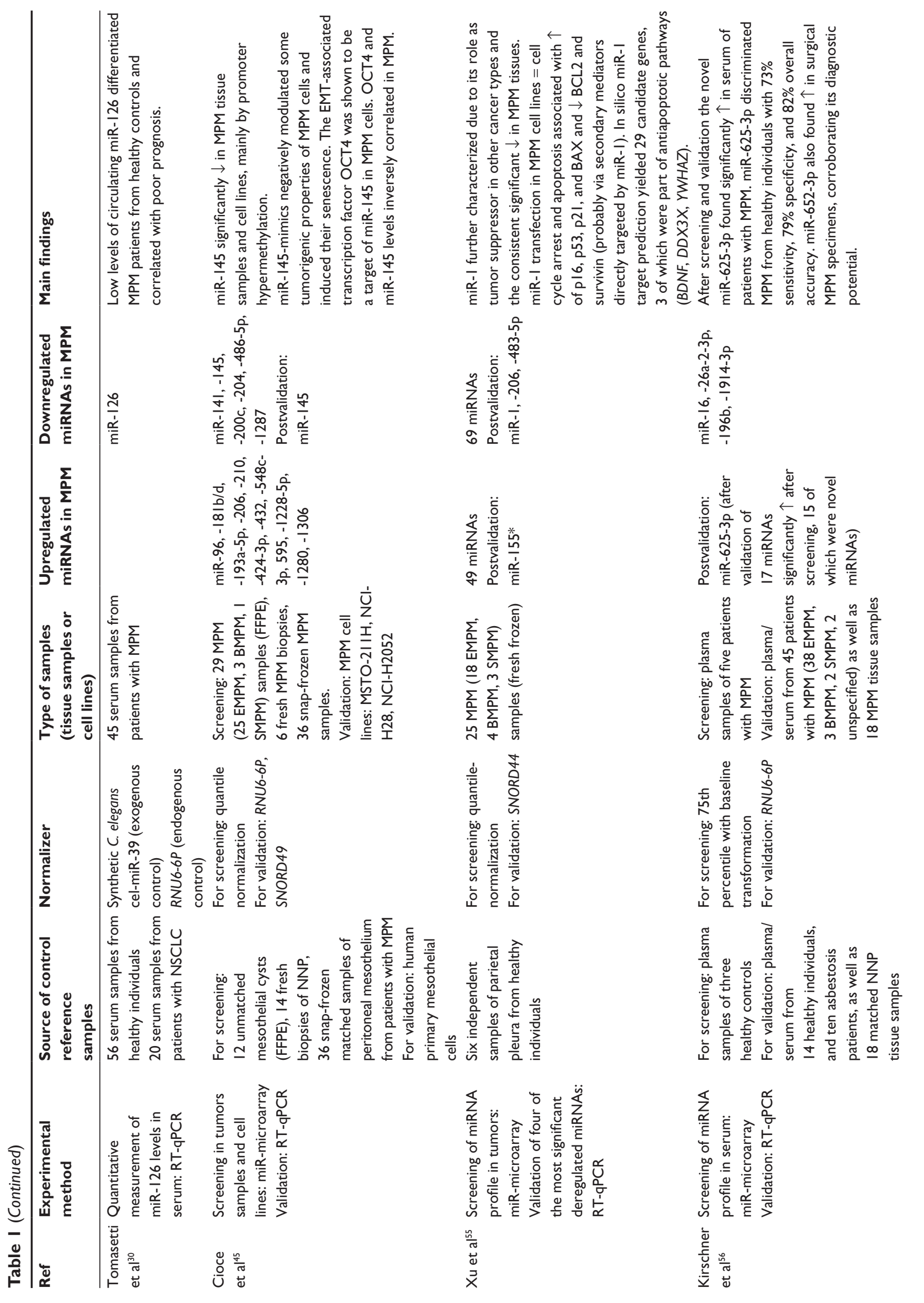



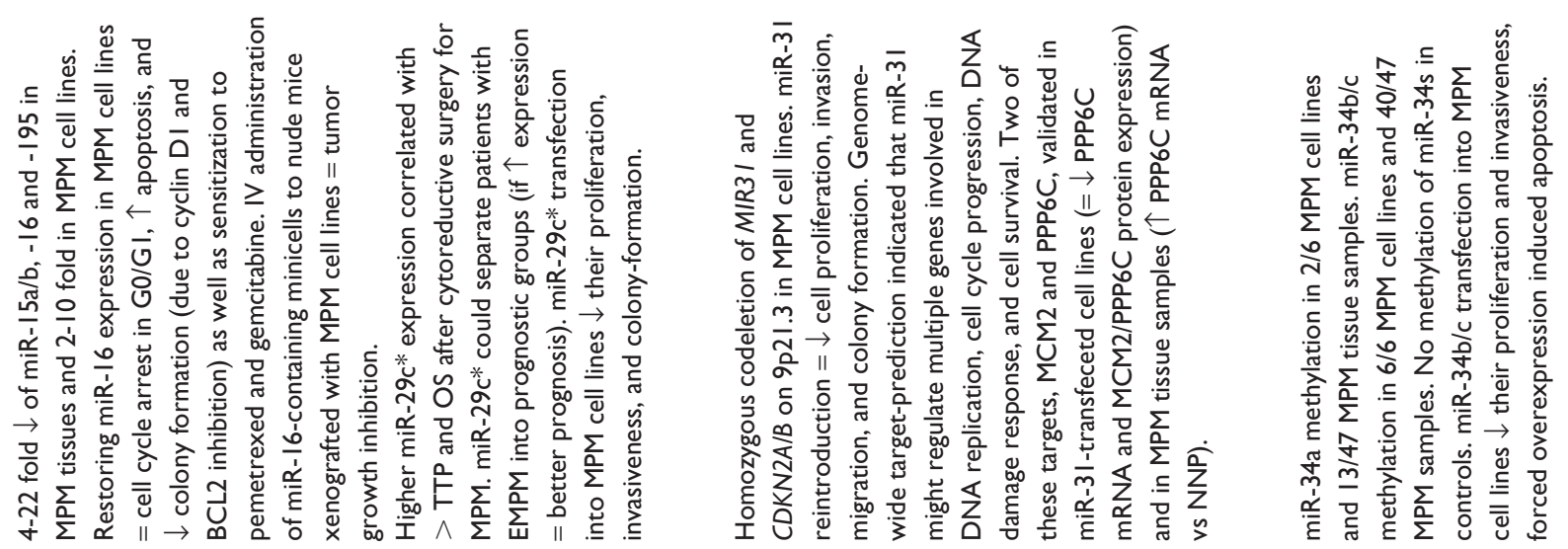

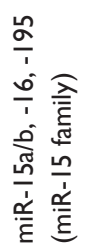

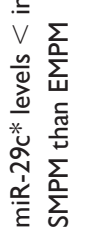

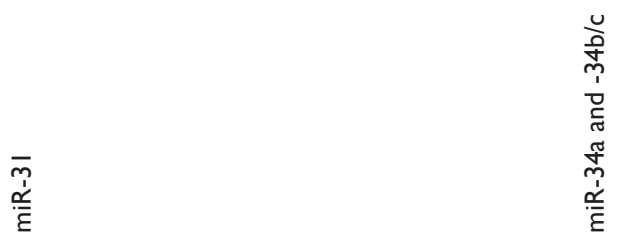

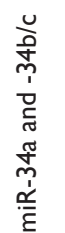

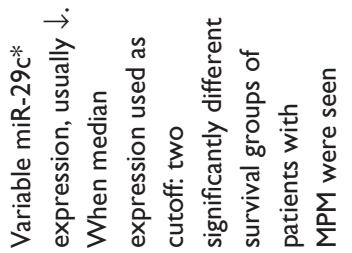

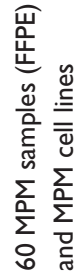

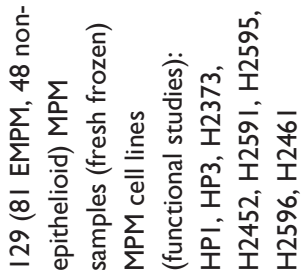

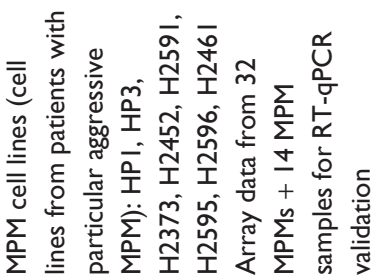

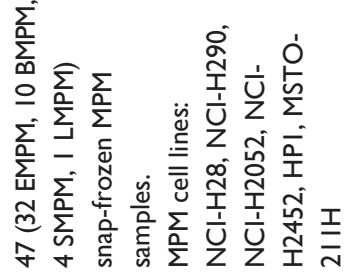
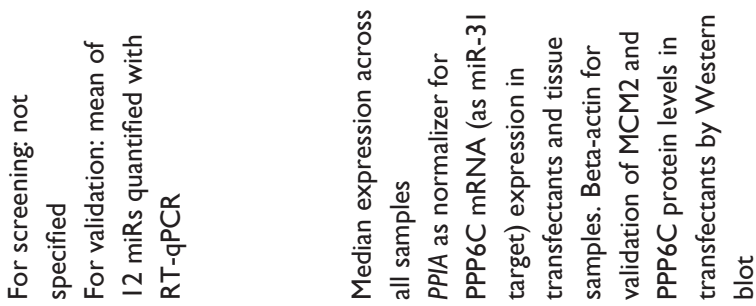

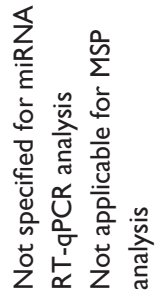

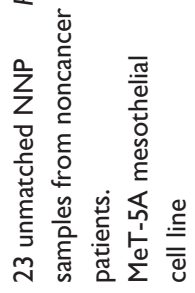

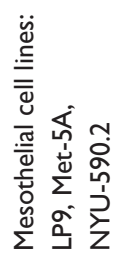
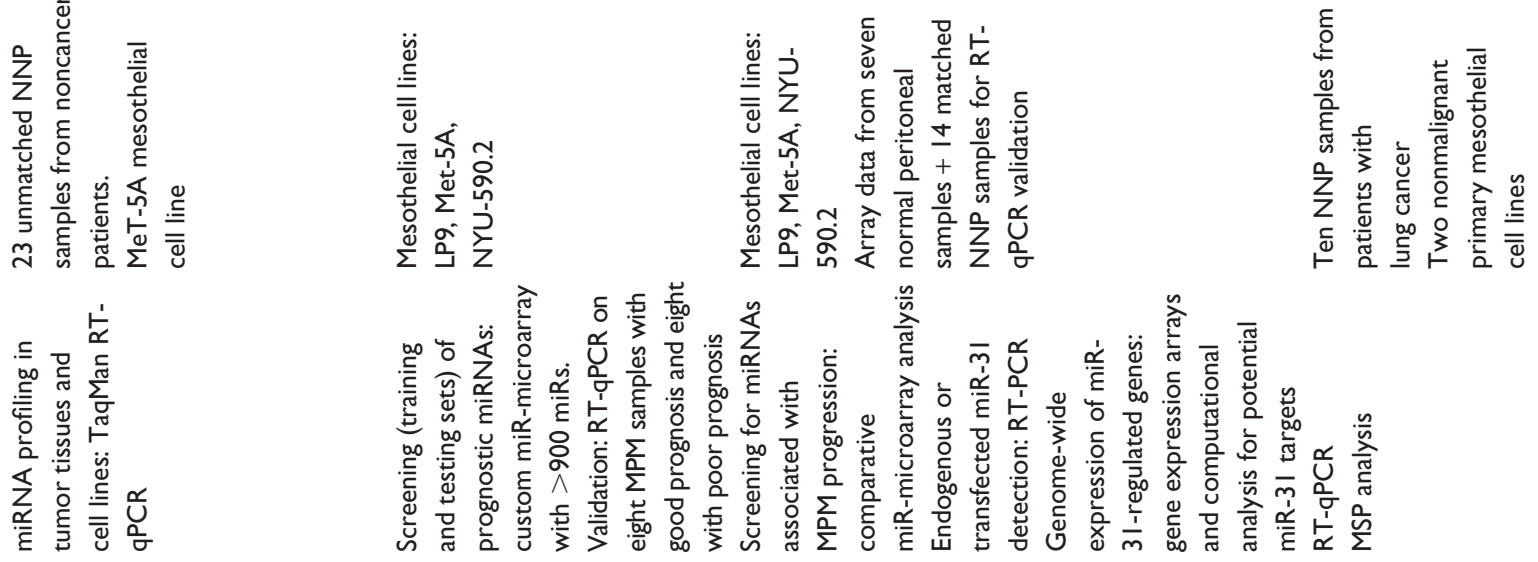

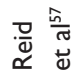

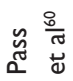

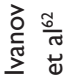

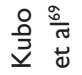




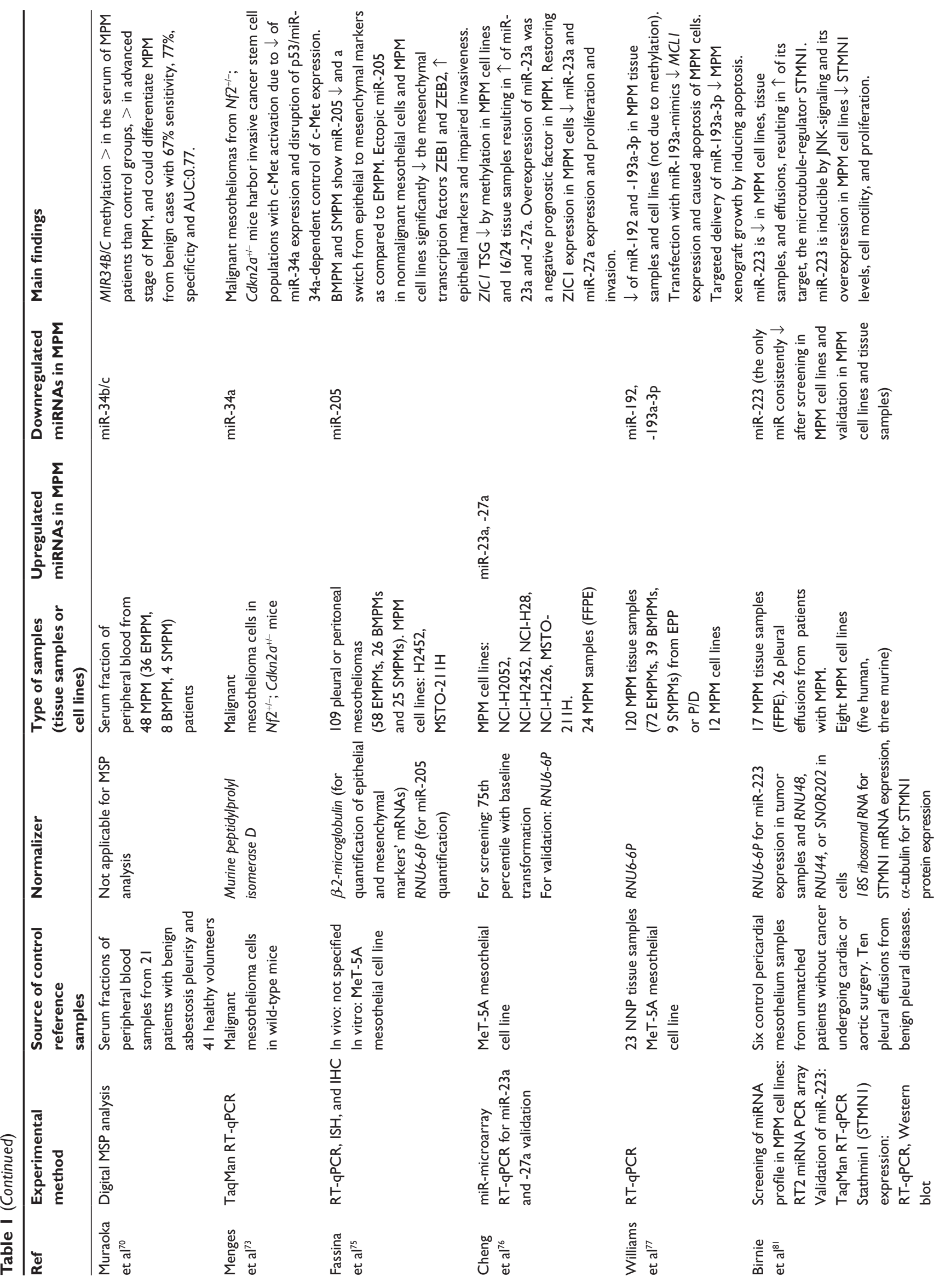




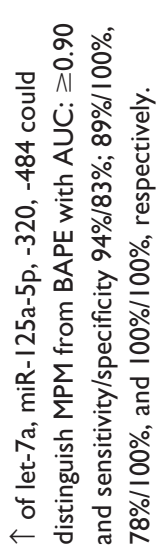

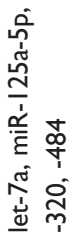

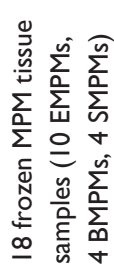

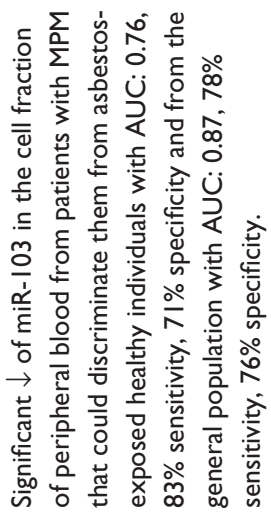

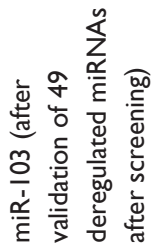



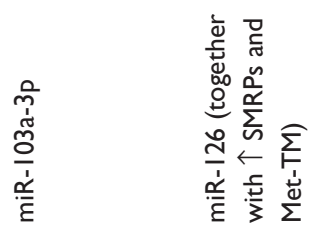

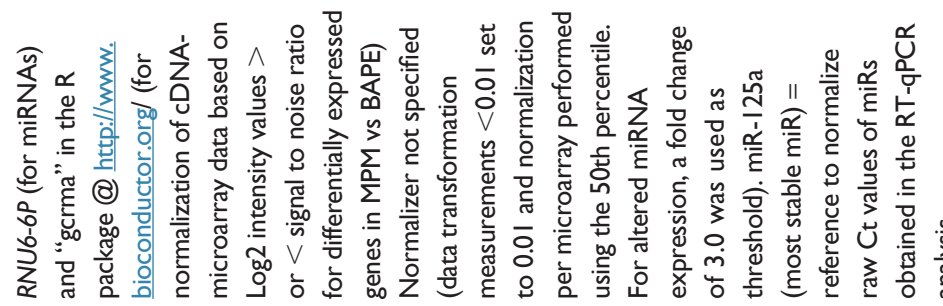
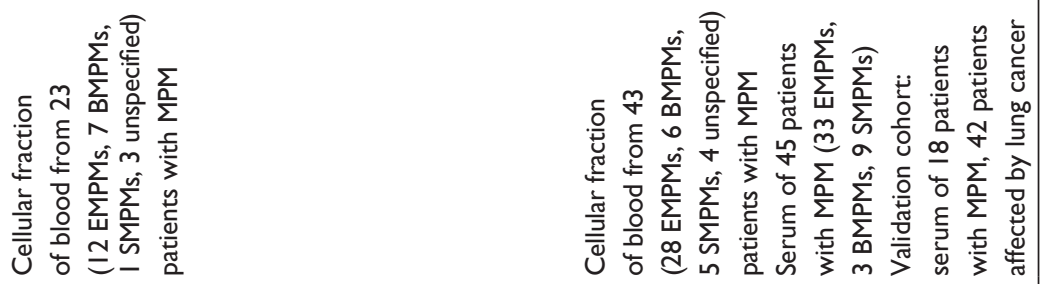
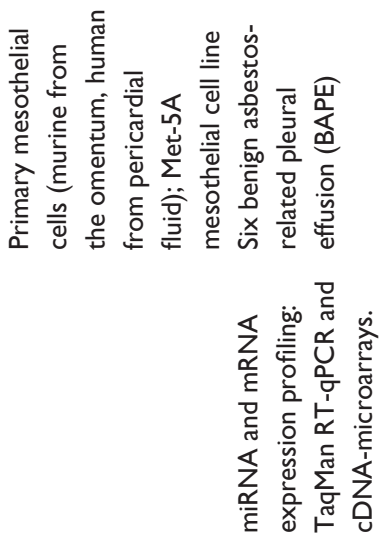

$\frac{\tilde{\omega}}{\frac{\tilde{\omega}}{\tilde{\omega}}}$
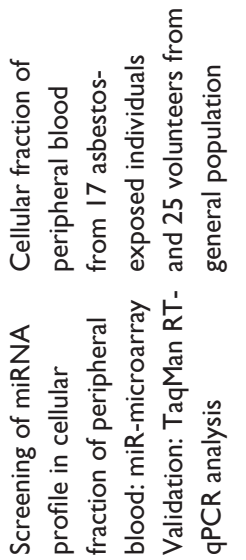

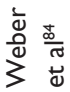
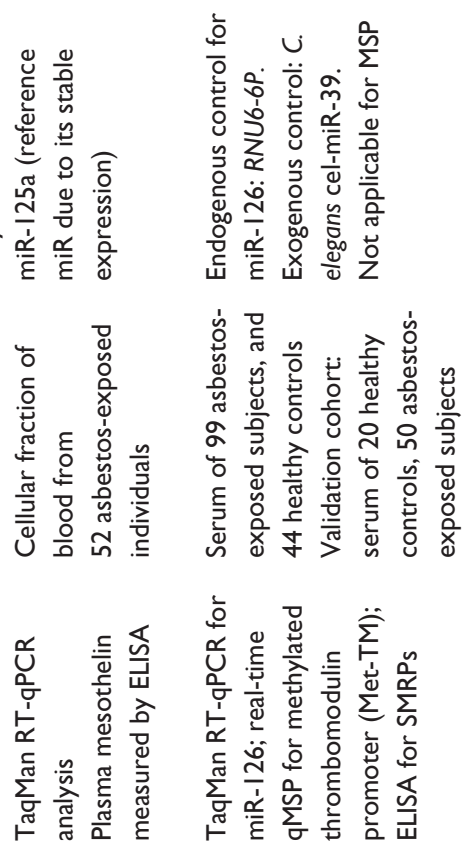

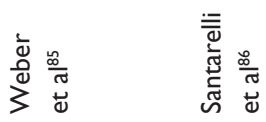




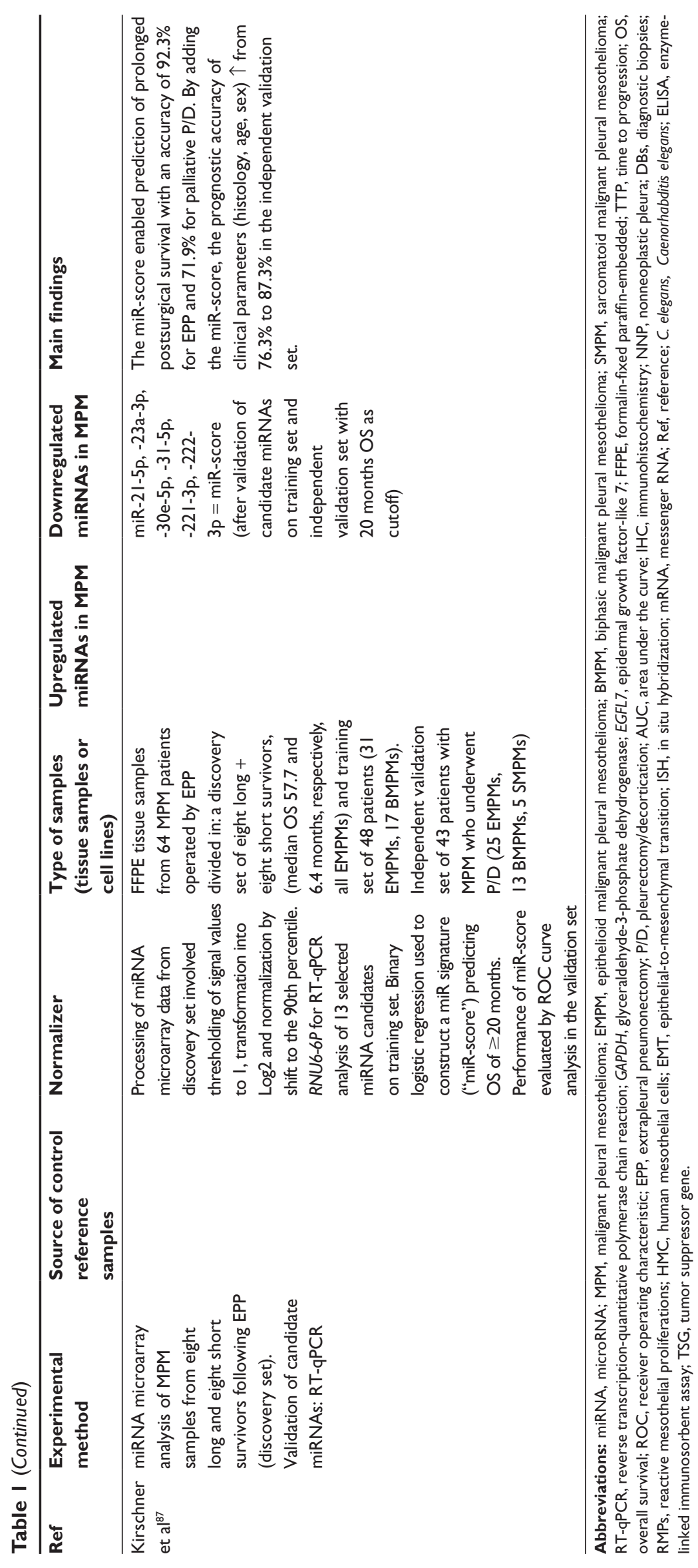


of miR-200 family members in the differential diagnosis between MPM and pulmonary adenocarcinoma. ${ }^{18}$ Using target prediction algorithms, furthermore, they identified multiple genes associated with the Wnt signaling pathway (eg, MYC and JUN) as putative targets of the downregulated miRNAs. ${ }^{18}$ However, with the exception of miR-17-5p and miR-106a overexpression in vitro, the aforementioned studies reported deregulation of different miRNAs in MPM cells and were difficult to compare. This is likely due to the differences in experimental setups, reference samples, and sources of specimens employed (MPM tissue samples vs cell lines) as well as size and goals of the studies (Table 1). Although the miRNA signatures obtained in these studies could apparently distinguish the different histological subtypes of MPM or discriminate between MPM cell lines and HMC or between MPM and other cancer types, it remained unclear whether the reported miRNAs could be useful as candidate biomarkers for the challenging differential diagnosis between MPM and reactive mesothelial proliferation (RMP) in patient samples.

Thus, we assessed whether the deregulation of miR17-5p, -30c, -221, and -222 observed in vitro may be useful in differentiating MPM from RMP in vivo. ${ }^{15,16,19}$ We quantified the expression of these four miRNAs by RT-PCR-based TaqMan $^{\circledR}$ assays in a set of patient-matched surgical FFPE samples of EMPM (stages I-IV) and nonneoplastic pleura (NNP) tissue from patients treated with trimodal protocol. ${ }^{19}$ We also included diagnostic biopsies from chemotherapynaïve patients with MPM and found no significant differential expression of these miRNAs in the diagnostic biopsies vs the surgical samples, suggesting that their expression was not affected by chemotherapy. Moreover, in contrast to the finding of Busacca et al in vitro, ${ }^{15}$ we found significant downregulation of miR-17-5p, upregulation of miR-221, and no differential expression of miR-30c and miR-222 in MPM when compared to NNP specimens. ${ }^{19}$ Thus, these four miRNAs appear differently regulated in MPM cells in vivo and in vitro. Suitable MPM marker should have a sensitivity or specificity of $>80 \%{ }^{1}{ }^{1}$ however, receiver operating characteristic curve analysis revealed that miR-17-5p and miR-221 did not fulfill these criteria (area under the curve [AUC] values of 0.64 and 0.65 , respectively). ${ }^{19}$ Therefore, despite being reportedly specific for MPM subtypes or differentially expressed in MPM cell lines vs HMC, miR-17-5p, miR-30c, and miR-221/222 do not seem to be the suitable biomarkers for accurately discriminating MPM from RMP.

Hence, to detect more specific miRNAs that could fulfill this task, we recently performed an RT-qPCR screening of
742 miRNAs and validated the 14 identified differentially expressed miRNAs with diagnostic potential in different cohorts of preoperative chemotherapy-naïve FFPE tumor biopsies, chemotherapy-treated surgically resected MPM specimens, and corresponding patient-matched NNP samples as well independent pleural samples from pneumothorax-induced RMPs. ${ }^{20}$ We discovered that miR$126,-143,-145$, and -652 were consistently downregulated in MPM resections and tumor biopsies and were the best candidate miRNAs to diagnose MPM. Not only these four miRNAs could significantly distinguish MPM from NNP/ RMP samples but combining by binary logistic regression analysis, the four miRNAs' expression data resulted in a 4-miR classifier with enhanced diagnostic performance that could correctly diagnose MPM with high sensitivity, specificity (AUC 0.96), and overall accuracy (94\%). Moreover, we showed that the 4-miR signature and high expression levels of miR-193b in MPM samples were associated with poor patient survival, suggesting that these miRNAs also possessed prognostic value. ${ }^{20}$ We also observed that miR126 expression in MPM was inversely correlated with the levels of one of its known targets, the large neutral amino acid transporter, small subunit 1 (LAT1), which was significantly overexpressed in MPM samples in comparison to the NNP and pneumothorax samples. ${ }^{20,21}$ Interestingly, our data on miR-126 downregulation's negative prognostic value and inverse correlation with LAT1 expression are consistent with the previously reported association between reduced survival and high LAT1 expression in MPM. ${ }^{22}$

\section{miRNAs in the diagnostic signature discriminating MPM from RMPs MicroRNA-I 26}

This miRNA has been reported downregulated in different malignancies. ${ }^{23-25}$ MIR126 is located within intron 7 of the EGFL7 gene, which encodes for epidermal growth factorlike 7 (EGFL7), an endothelial cell-secreted protein that contributes to normal and pathological vasculogenesis. ${ }^{26,27}$ $E G F L 7$ contains eleven introns generating three different transcripts through three independent transcriptional initiation sites. MIR126 is coexpressed with one of these alternative transcripts which has transcription initiation site positioned at a $\mathrm{CpG}$ island in intron 2 of $E G F L 7 .{ }^{27}$ Aiming at elucidating the molecular mechanisms of the downregulation of miR-126 in MPM, we recently discovered that miR-126 and its host EGFL7 gene were cosilenced in MPM by hypermethylation of $\mathrm{CpG}$ islands flanking EGFL7's transcription-start-site in intron 2. This hypermethylation of EGFL and MIR126 was 
Table 2 Predicted or verified target genes of deregulated miRNAs in MPM

\begin{tabular}{|c|c|c|c|}
\hline Ref & miRNA(s) & Target gene(s) & Evidence \\
\hline Guled et al ${ }^{14}$ & miR-885-3p & CDKN2A, NF2 & Computational \\
\hline Guled et al ${ }^{14}$ & miR-7-I* & EGF, PDGFA & Computational \\
\hline Guled et al ${ }^{14}$ & miR-30b*, $-32 *,-483-3 p,-584$ & $R B I$ & Computational \\
\hline Guled et al ${ }^{14}$ & miR-203 & HGF, CTNNBIPI, DKKI, DKK4, DVL3, & Computational \\
\hline Gee et al ${ }^{18}$ & & EGRI, FZD2, NLK, WNT8A & \\
\hline Guled et al ${ }^{14}$ & miR-9, - I4I, -200a*/b/c, -203, -429 & JUN & Computational \\
\hline \multirow[t]{4}{*}{ Gee et al ${ }^{18}$} & (certain targets are predicted to be & & \\
\hline & regulated by $-200 \mathrm{a} * / 14 \mathrm{I}$ and $-200 \mathrm{~b} /$ & & \\
\hline & c/429. These possibilities also concern & & \\
\hline & the other putative targets underneath) & & \\
\hline Busacca et $\mathrm{al}^{15}$ & miR-17-5p, - I06a, - 143 & BTGI & Computational \\
\hline Balatti et al ${ }^{16}$ & miR-17-5p, -20a, -92 & CDKNIA & Western blot \\
\hline Gee et $\mathrm{al}^{18}$ & miR-203, -205 & $A P C$ & Computational \\
\hline Gee et $\mathrm{al}^{18}$ & $\operatorname{miR}|4|,-200 a^{*}$ & CTNNBI, DKK3 & Computational \\
\hline Gee et $\mathrm{al}^{18}$ & miR-I4I, 200a*/b/c, -203, -429 & $D I X D C I$ & Computational \\
\hline Gee et $\mathrm{al}^{18}$ & $\operatorname{miR} \mid 4 I,-200 a^{*},-203$ & $D K K 2$ & Computational \\
\hline Gee et $\mathrm{al}^{18}$ & miR-I4I, -200a* & SOXI7 & Computational \\
\hline Gee et $\mathrm{al}^{18}$ & $\mathrm{miR}-200 \mathrm{~b} / \mathrm{c},-429$ & EP300 & Computational \\
\hline Gee et $\mathrm{al}^{18}$ & $m i R-I 4 I,-200 a * / b / c,-429$ & $F B X W 2, F B X W I I$ & Computational \\
\hline Gee et $\mathrm{al}^{18}$ & miR-200a* & FGF4 & Computational \\
\hline Gee et $\mathrm{al}^{18}$ & miR-I4I, -200a*, -203, -205 & FSHB & Computational \\
\hline Gee et $\mathrm{al}^{18}$ & miR-200a*, -203, -205 & FZDI & Computational \\
\hline Gee et $\mathrm{al}^{18}$ & miR-|4I, $-200 \mathrm{a} * / \mathrm{b} / \mathrm{c} /,-203,-429$ & FZD4 & Computational \\
\hline Gee et $\mathrm{al}^{18}$ & $\mathrm{miR}-200 \mathrm{~b} / \mathrm{c},-203,-429$ & FZD5, FZD6 & Computational \\
\hline Gee et $\mathrm{al}^{18}$ & $m i R-|4|,-200 a^{*}$ & FZD8, SOXI7 & Computational \\
\hline Gee et $\mathrm{al}^{18}$ & miR-I4I, -200a*, -205 & LRP6 & Computational \\
\hline Gee et $\mathrm{al}^{18}$ & $\mathrm{miR}-200 \mathrm{~b} / \mathrm{c},-203,-429$ & MYC & Computational \\
\hline Gee et $\mathrm{al}^{18}$ & $\mathrm{miR}-200 \mathrm{~b} / \mathrm{c},-205,-429$ & NKDI & Computational \\
\hline Gee et $\mathrm{al}^{18}$ & miR-|4I, -200a*, -203, -205 & PLCBI & Computational \\
\hline Gee et $\mathrm{al}^{18}$ & miR-I4I, $-200 \mathrm{a} / \mathrm{b} / \mathrm{c},-429$ & PPP2CA & Computational \\
\hline Gee et $\mathrm{al}^{18}$ & miR-I4I, $-200 \mathrm{a} * / \mathrm{b} / \mathrm{c} /,-203,-429$ & PPP2R5C, PPP2R5E & Computational \\
\hline Gee et $\mathrm{al}^{18}$ & $\mathrm{miR}-200 \mathrm{~b} / \mathrm{c},-429$ & RHOA, ROCK2 & Computational \\
\hline Gee et $\mathrm{al}^{18}$ & miR-I4I, -200a*/b/c/, -203, -205, -429 & TCF4 & Computational \\
\hline Gee et $\mathrm{al}^{18}$ & miR-|4I, $-200 \mathrm{a} * / \mathrm{b} / \mathrm{c} /,-203,-429$ & VANGLI & Computational \\
\hline Gee et $\mathrm{al}^{18}$ & $\mathrm{miR}-\mathrm{I} 4 \mathrm{I},-200 \mathrm{~b} / \mathrm{c} /,-203,-205,-429$ & VEGFA & Computational \\
\hline Gee et $\mathrm{al}^{18}$ & $\mathrm{miR}-200 \mathrm{~b} / \mathrm{c},-203,-429$ & WIFI, WNT4 & Computational \\
\hline Gee et $\mathrm{al}^{18}$ & miR-205 & WNT3A, WNT5B & Computational \\
\hline Gee et $\mathrm{al}^{18}$ & $m i R-|4|,-200 a * / b / c,-429$ & WNT5A, WNTI6 & Computational \\
\hline Andersen et $\mathrm{al}^{20}$ & miR-I26 & LATI & $\begin{array}{l}\text { IHC with } H \text {-score, Spearman test for } \\
\text { correlation between miR-I } 26 \text { and LATI } \\
\text { expression }\end{array}$ \\
\hline Bao et $\mathrm{al}^{34}$ & miR-I26 & IRSI & $\begin{array}{l}\text { Luciferase assay, transfection with MIR/26 } \\
\text { expression plasmid and pGL3-promoter } \\
\text { plasmid containing IRSI } 3^{\prime} \text {-UTR }\end{array}$ \\
\hline Cioce et $\mathrm{a}^{45}$ & miR-I45 & OCT4 & Computational, luciferase activity, Western \\
\hline & & & blot, miR-I45-mimic transfection \\
\hline Xu et $a^{55}$ & miR-I & $\begin{array}{l}\text { ANKIBI, ARID2, AZINI, BCLIIA, BDNF, } \\
\text { CALM2, CLTC, DDX3X, EFNB2, FBXW7, } \\
\text { FNDC3A, FNDC3B, HAND2, HNRNPU, } \\
\text { HS3ST3BI, JARID2, MIPOLI, MON2, } \\
\text { NCL, NR4A2, PICALM, PTMA, RNFI65, } \\
\text { TBCIDI5, TMSL3, VEZFI, YWHAZ, } \\
\text { ZFHX4, ZNF827 }\end{array}$ & Computational \\
\hline Reid et al ${ }^{57}$ & miR-16 & $\begin{array}{l}\text { Many predicted and qPCR-validated } \\
\text { targets, of these CCNDI and BCL2 also } \\
\text { validated at protein level }\end{array}$ & $\begin{array}{l}\text { miR-16-mimic transfection, RT-qPCR, } \\
\text { Western blot }\end{array}$ \\
\hline Pass et $\mathrm{al}^{60}$ & $\operatorname{miR}-29 c^{*}$ & ADIPOQ, CTRP8, CTRPI, DNMT3A & Transfection of miR-29c*-mimic, RT-qPCR \\
\hline
\end{tabular}


Table 2 (Continued)

\begin{tabular}{|c|c|c|c|}
\hline Ref & miRNA(s) & Target gene(s) & Evidence \\
\hline Ivanov et $\mathbf{a}^{62}$ & miR-3I & $\begin{array}{l}\text { Computational target prediction: } \\
\text { multiple genes, of which CCNB2, CDC2, } \\
\text { CDC6, CHEKI, SKP2 most } \uparrow \text { in MPM. } \\
\text { Predicted and validated: MCM2, PPP6C }\end{array}$ & $\begin{array}{l}\text { miR-3I transfection, gene expression array, } \\
\text { computational search, RT-qPCR in miR-3I- } \\
\text { transfected MPM cell lines and in MPM tissue } \\
\text { samples, Western blot in transfectants }\end{array}$ \\
\hline $\begin{array}{l}\text { Kubo et al, }{ }^{69} \\
\text { Maki et al }{ }^{71}\end{array}$ & miR-34b, $-34 c$ & $\begin{array}{l}\text { BCL2, CCNDI, CCNE2, CDK4, CDK6, } \\
\text { E2F3, MET, MYC }\end{array}$ & $\begin{array}{l}\text { miR-34b/c transfection in MPM cell lines, } \\
\text { Western blot }\end{array}$ \\
\hline Tanaka et al ${ }^{72}$ & miR-34a, $-34 b,-34 c$ & $B C L 2, M E T$ & $\begin{array}{l}\text { Transfection of miR-34 inhibitors in HMC, } \\
\text { Western blot }\end{array}$ \\
\hline Menges et $\mathrm{al}^{73}$ & miR-34a & MET & $\begin{array}{l}\text { siRNA silencing of miR34 in } \mathrm{N}^{2+-} ; \mathrm{Cdkn} 2 \mathrm{a}^{+/-} \\
\text {mice }\end{array}$ \\
\hline Fassina et $\mathrm{al}^{75}$ & $\operatorname{miR}-205$ & ZEBI, ZEB2 & $\begin{array}{l}\text { miR-205 transfection in nonmalignant } \\
\text { mesothelial cells and MPM cell lines, } \\
\text { RT-qPCR }\end{array}$ \\
\hline Williams et $\mathrm{al}^{77}$ & miR-193a-3p & E2FI, MCLI, TYMS & RT-qPCR, Western blot \\
\hline Birnie et $a^{81}$ & miR-223 & STMNI & $\begin{array}{l}\text { RT-qPCR, Western blot, } \\
\text { immunoprecipitation }\end{array}$ \\
\hline Ak et $\mathrm{al}^{82}$ & miR-449a & CCNE2, MET & $\begin{array}{l}\text { Integrated analysis of } \uparrow \text { mRNAs and target } \\
\text { genes of } \downarrow \text { miRNAs }\end{array}$ \\
\hline Kirschner et al ${ }^{87}$ & $\begin{array}{l}\text { miR-2I-5p, -23a-3p, -30e-5p, -3I-5p, } \\
-22 \text { I-3p, -222-3p (prognostic } 6 \\
\text { miR-signature = "miR-score") }\end{array}$ & $\begin{array}{l}\text { Multiple genes associated to pathways } \\
\text { implicated in MPM development } \\
\text { (Wnt-, Hippo-, FAK-, PI3K/Akt-, } \\
\text { ErBb-signaling, etc) }\end{array}$ & $\begin{array}{l}\text { Computational pathway enrichment analysis } \\
\text { of miR-score's miRNA target genes }\end{array}$ \\
\hline
\end{tabular}

Abbreviations: miRNA, microRNA; mRNAs, messenger RNAs; MPM, malignant pleural mesothelioma; IHC, immunohistochemistry; IRSI, insulin receptor substrate- I; CCNDI, cyclin DI-encoding gene; miR-I26, MicroRNA-I26; RT-qPCR, reverse transcription-quantitative polymerase chain reaction; HMC, human mesothelial cell; Ref, reference.

predominantly associated with epithelioid histology and correlated with poor clinical outcome. ${ }^{28}$ These results suggest that demethylating strategies reestablishing EGFL7 and miR-126 expression might provide future therapeutic options for MPM. Moreover, considering that miR-126 has promising diagnostic potential in MPM, ${ }^{20}$ concomitant silencing of EGFL7 might represent an additional event that could be used in the difficult differential diagnosis between MPM and RMPs.

Downregulation of miR-126 was first reported in MPM by Santarelli et al after expression screening of 88 cancerrelated miRNAs in an independent set of fresh-frozen MPM biopsies, surgical FFPE MPM samples, and adjacent NNP. ${ }^{29}$ These authors also reported that the serum levels of miR-126 were reduced in patients with MPM and subjects who are at high risk to develop MPM and were inversely correlated with the circulating soluble mesothelin-related protein (SMRP) concentration. Thus, circulating miR-126 and SMRP were proposed as valuable biomarkers for the early diagnosis of MPM. ${ }^{29}$ Confirming the relevance of these findings, low levels of circulating miR-126 differentiated patients with MPM from healthy control subjects and correlated with poor prognosis. ${ }^{30}$ By targeting genes such as $L A T 1$, vascular endothelial growth factor-A (VEGF-A), EGFL7 itself (as a feed back), insulin receptor substrate-1 (IRS1), phosphoinositide-3-kinase, regulatory subunit 2 (PI3KR2), and Kirsten rat sarcoma viral oncogene homolog $(K R A S)$, miR-126 has been linked to vascular integrity and tumor suppression..$^{20,21,31-33}$ However, the mechanisms by which miR-126 suppresses mesothelioma genesis remain poorly understood. Recently, Tomasetti et al showed that ectopic miR-126 modified the response of the MPM cell line NCI-H28 to hypoxic oxidative stress through the inhibition of IRS1-PI3K-Akt signaling and activation of the Forkhead-boxO1 (FoxO1) transcription factor, resulting in mitochondrial respiration repression, increased glycolysis, and FoxO1-induced gluconeogenesis and oxidative stress defense. ${ }^{34}$ The authors also observed concomitant activation of the hypoxia-inducible factor- $1 \alpha$, which controls the adaptation of malignant cells to hypoxic microenvironments and the expression of glucose transporters, glycolytic enzymes, angiogenic growth factors, and apoptosis regulators. ${ }^{34,35}$ Moreover, the expression of miR126 in NCI-H28 cells suppressed their proliferation, colony formation, and tumor formation in nude mice. ${ }^{34}$ Thus, miR126 appears to be tumor suppressive in MPM at least in part through pleiotropic metabolic effects linked to the inhibition of the IRS1-PI3K-Akt signaling pathway.

It has also been shown that miR-126 targets certain chemokines and related receptors, which are highly expressed in MPM and promote the accumulation of lymphocytes and mesenchymal stem cells. Thus, changes in the tumor microenvironment due to miR-126 downregulation may in turn favor cancer progression by enhancing invasiveness and metastatic potential of neoplastic cells. ${ }^{36-38}$ 


\section{MicroRNA-I43 and - I 45}

miR-145 is clustered with miR-143 and often lost in several cancer types. ${ }^{39-41}$ The expression of miR-143/-145 cluster is regulated by $\mathrm{p} 53$, and the oncogene $M D M 2$ is a direct target of miR-143/-145; thus, deregulation of miR-143/-145 in malignant cells impairs the MDM2-p53 feedback loop. ${ }^{42}$ Additionally, miR-143 and miR-145 are also considered tumor suppressors because they target other oncogenes..$^{40-44}$ Further studies assessing the expression of miR-143 and miR145 together with that of some of their targets will clarify whether these regulatory loops are also relevant for MPM.

A recent study comparing the expression of 887 miRNAs in FFPE MPM-specimens and a control group of unmatched mesothelial cysts showed that miR-145 was the most deregulated among 19 differentially expressed miRNAs. ${ }^{45}$ The downregulation of miR-145 in malignant mesothelial cells was validated in independent sets of unmatched MPM and NNP biopsies, patient-matched peritoneal mesothelioma and normal mesothelium, and MPM cell lines as compared to HMC. These results, together with our findings, ${ }^{20}$ indicate that the expression levels of miR-145 can be potentially useful for differentiating benign from malignant mesothelial proliferations. Moreover, downregulation of miR-145 in malignant mesothelial cells was in large part due to MIR145promoter hypermethylation. ${ }^{45}$ Similarly, we recently found that, in contrast to patient-matched NNP samples, MPM specimens display methylated or hemimethylated MIR145 gene promoter (Aslan et al, unpublished data, 2015).

From a mechanistic point of view, forced expression of miR-145-agonists in MPM cell lines impaired their proliferation, colony formation, migration, and resistance to pemetrexed treatment, leading to replicative senescence and hampering the ability of cells to form tumors after transplantation into SCID mice. ${ }^{45}$ Furthermore, miR-145 targets the transcription factor OCT4, which regulates the epithelial-to-mesenchymal transition (EMT), and has been associated with acquired resistance to pemetrexed in MPM. ${ }^{45,46}$ Accordingly, ectopic miR-145 downregulated OCT4 in MPM cell lines, while reintroduction of OCT4 into these cells counteracted miR-145-induced replicative senescence. Consistent with these observations, an inverse correlation between the levels of miR-145 and OCT4 was observed in MPM samples. ${ }^{45}$ Collectively, these data imply that miR-145 acts as mesothelial tumor suppressor at least in part by targeting OCT4.

\section{MicroRNA-652}

In contrast to the tumor-suppressive miR-126, -143, and -145 , miR-652 is poorly characterized. Single reports have described deregulated miR-652 expression in other tumor types. ${ }^{47,48}$ Diagnostic and prognostic potentials of miR-652 deregulation have been reported in breast cancer. ${ }^{49,50}$ However, the knowledge on validated targets and mechanism of action for miR-652 is scant. This miRNA was recently found upregulated in the dysfunctional heart, and the hematopoiesis-related Notch1-ligand, Jagged1, was identified and validated as miR-652 target in a mouse cardiac hypertrophy model. ${ }^{51}$ Notably, Jagged 1 was previously found overexpressed in MPM cell lines. ${ }^{52}$ To learn more about potential miR-652 targets and determine specific pathways possibly regulated by miR-652, we recently performed in silico computational analysis combining seven different target prediction algorithms and functional annotation tools. Preliminary unpublished data show that miR-652 may target genes encoding proteins of cellular membranes involved in the regulation of transport activities of ion channels or acting as receptors. Interestingly, among these putative miR-652 targets, we found the $B S G$ gene encoding basigin/CD147, a membrane protein that has been related to metastatic potential, drug resistance, and poor prognosis in different cancer types and has been proposed as IHC-marker for distinguishing benign RMP cells from MPM. ${ }^{53,54}$ Further investigations are needed to corroborate these data and expand the knowledge of miR-652 function in MPM.

\section{Role of other deregulated miRNAs with diagnostic or prognostic value in MPM}

Additional studies have shed light on the role of pathophysiologic, diagnostic, prognostic as well as therapeutic relevance of individual miRNAs or families of miRNAs in MPM.

\section{MicroRNA-I}

$\mathrm{Xu}$ et al reported significant downregulation of miR-1 in 25 MPM-specimens (18 EMPM, four BMPM, and three SMPM), as compared to six pleura samples from patients with out cancer. ${ }^{55}$ miR-1 transfection in MPM cell lines caused cell cycle arrest and apoptosis associated with induction of the tumor suppressors $\mathrm{p} 53$, BCL2-associated $\mathrm{X}$ protein, and cyclin-dependent kinase inhibitors p16 and p21 as well as repression of BCL2 and survivin, possibly through secondary mediators directly targeted by miR- $1 .{ }^{55}$ Further corroboration in vivo is required in order to completely evaluate pathogenetic and therapeutic significance of miR-1 in MPM.

\section{MicroRNA-16}

The underexpression of miR-16, a member of the miR15 family, which according to the miRBase comprises 
miR-15a, -15b, -16, and $-195,{ }^{10}$ was first reported in MPM by Kirschner et al. ${ }^{56}$ Subsequently, Reid et al found marked underexpression of the entire miR-15 family (fourfold to 22-fold) when they compared a set of 60 FFPE MPM specimens to a reference cohort of 23 NNP specimens from patients undergoing cardiac or aortic surgery. ${ }^{57}$ Similar downregulation (twofold to tenfold) of miR-15 family was detected in MPM cell lines. ${ }^{57}$ Restoring miR-16 expression in these cell lines resulted in cell cycle arrest in G0/G1, increased apoptosis, and reduced colony formation, consistent with the cyclin D1-encoding gene (CCND1) and the antiapoptotic gene $B C L 2$ being known miR-16 targets. ${ }^{57-59}$ Transfecting varying doses of miR-16-mimics resulted also in dose-dependent sensitization to pemetrexed and gemcitabine, while IV administration of miR-16-containing minicells to nude mice xenografted with MPM cell lines led to significant dose-dependent tumor growth inhibition. ${ }^{57}$ Together, these results imply that miR-16 may have diagnostic potential and reestablishing its expression may represent a potential future therapeutic tool for MPM.

\section{MicroRNA-29c*}

Investigating the prognostic impact of specific miRNAs in a cohort of surgically treated patients with MPM, Pass et al discovered that the expression of miR-29c* correlated with time-to-progression and overall survival after cytoreductive surgery. ${ }^{60}$ The levels of this miRNA were higher in EMPM than in SMPM and could be used to separate patients with epithelioid histology into prognostic groups, as increased expression was associated with more favorable prognosis. ${ }^{60}$ miR-29c* transfection of MPM cell lines decreased their proliferation, invasiveness, and colony formation, suggesting an antitumorigenic function of this miRNA as well. Moreover, the levels of DNA methyltransferases in MPM were found to be more elevated than in corresponding NNP, while the miR-29c*-transfected cell lines displayed significant downregulation of these enzymes and reexpression of specific methylated genes. ${ }^{60}$ This is consistent with previous data showing that the DNA-(cytosine-5)-methyltransferases DNMT3A and DNMT3B are targets of the miR-29 family members (miR-29a/b/c) ${ }^{61}$

\section{MicroRNA-3I}

Deletion of the $9 \mathrm{p} 21.3$ chromosomal region, including the TSGs $C D K N 2 A / 2 B$, is frequent in MPM and is associated with poor prognosis. ${ }^{1}$ Intriguingly, the $9 \mathrm{p} 21.3$ region harbors the MIR31 gene, which is frequently codeleted with the CDKN2 genes in MPM cell lines derived from rapidly progressing MPMs. Moreover, reintroduction of miR-31 inhibits the proliferation, invasion, migration, and clonogenicity of these cells. ${ }^{62}$ A genome-wide search using target-prediction algorithms indicated that miR-31 might regulate multiple genes involved in DNA replication, cell cycle progression, DNA damage response, and cell survival (Table 2). ${ }^{62}$ One particularly interesting putative miR-31 target is the mRNA for the catalytic subunit of the prosurvival protein phosphatase 6 (PPP6C). This transcript, which contains three binding sites for miR-31 in its 3'-UTR region, was repressed in miR-31-transfected MPM cells and overexpressed in miR-31-deleted MPM specimens. ${ }^{62}$ In addition to providing survival advantages, PPP6C is involved in the maintenance of DNA replication, chromosomal segregation, and cell cycle progression as well as resistance to chemoradiotherapy; thus, its overexpression in MPM may result in aberrant control of these fundamental cellular processes. ${ }^{62,63}$ Together, these results suggest that the deletion of miR-31 and overexpression of PPP6C may be potential diagnostic biomarkers for MPM. Moreover, the reintroduction of miR-31 or the inhibition of its targets might help to recoordinate cell division with DNA repair and chromosomal stability, thereby representing possible future therapeutic avenues to pursue in MPM.

Another group recently reported significant downregulation of miR-31 in FFPE MPM vs RMP samples. ${ }^{64}$ However, four out of the five cases with higher miR-31 levels were BMPM or SMPM and associated with worse prognosis when compared to BMPM or SMPM with lower miR-31 expression. ${ }^{64}$ Although the data support a possible role for miR-31 in distinguishing MPM from benign RMPs, its value in differentiating the histological subtypes of MPM and its prognostic impact require analysis of larger cohorts.

\section{The microRNA-34 family}

This miRNA family includes miR-34a, encoded by the MIR34A gene on chromosome 1p36.22 and miR-34b/-34c, which derive from a single primary transcript of MIR34B/ MIR34C on chromosome 11q23.1. The miR-34s are p53 transcriptional targets; mediate some of tumor-suppressive p53-functions; and regulate cell proliferation, survival, invasion, and angiogenesis. ${ }^{65-68}$ Epigenetic silencing of miR-34s appears to be involved in mesothelioma genesis. Kubo et al detected the methylation of the MIR34A promoter region in two of the six tested MPM cell lines and 13 of 47 resected MPM samples (of different histotypes), whereas the $M I R 34 B / C$ promoter was methylated in all six cell lines and in 40 of 47 tumor samples. ${ }^{69}$ Methylation correlated with the reduced expression of miR-34s. In contrast, no methylation of $M I R 34 A / B / C$ was detected in two nonmalignant mesothelial cell cultures or ten NNP samples surgically removed from patients with lung cancer. ${ }^{69}$ Reestablishing the 
physiologic miR-34b/c expression levels in MPM cell lines by stable transfection suppressed cell proliferation, -migration, and -invasion, while the overexperssion of $\mathrm{miR}-34 \mathrm{~b} / \mathrm{c}$ by adenoviral vectors led to apoptotic cell death. ${ }^{69}$

In keeping with these results, circulating $M I R 34 B / C$-DNA was observed to be significantly more methylated in the serum of 48 patients with MPM than 21 patients with benign asbestos pleurisy and 41 healthy volunteers. ${ }^{70}$ Advanced MPM cases displayed more methylation than early cases, and receiver operating characteristic curve analysis showed that $M I R 34 B / C$ methylation could differentiate MPM from benign cases with sensitivity of $67 \%$, specificity of $77 \%$, and AUC of $0.77 .{ }^{70}$

Furthermore, miR-34b/c enhanced the radiosensitivity of MPM cell lines by impairing DNA repair potential after X-ray irradiation. ${ }^{71}$ In contrast, inhibiting $\mathrm{miR}-34 \mathrm{a} / \mathrm{b} / \mathrm{c}$ expression with complementary RNA oligonucleotides in primary HMC significantly increased their proliferation, migration, and colony formation. ${ }^{72}$ Upregulation of the c-Met tyrosine-kinase receptor and the antiapoptotic effector BCL2 was detected in the oligonucleotide-transfected normal mesothelial cells, ${ }^{72}$ consistent with their reported pathogenetic role as potential $\mathrm{miR}-34 \mathrm{~b} / \mathrm{c}$ targets in MPM (Table 2). ${ }^{69}$

The impact of miR-34-mediated c-Met-regulation was further confirmed in vivo. Asbestos-exposed mice with heterozygous deletion of the TSGs $N F 2$ and CDKN2A showed accelerated mesothelioma genesis characterized by highly invasive and metastatic cells, including populations of cancer stem cells with c-Met activation. ${ }^{73}$ Notably, this c-Met activation was partly dependent on disrupted p53-mediated miR-34a induction. Thus, the inactivation of the p53/miR34a-dependent c-Met-modulation appears to be an important step in the development of MPM associated with the loss of NF2 and CDKN2A.73

Collectively, the aforementioned studies suggest that the methylation and downregulation of miR-34s may have diagnostic potential in MPM and restoring their expression could represent a future appealing therapeutic option in this dismal disease. Evaluating this concept preclinically, Ueno et al subcutaneously transplanted the miR-34-methylated MPM cell line NCI-H290 into BALB/C mice and observed that the resulting tumor growth was significantly inhibited by injecting a miR-34b/c-expressing adenoviral vector. ${ }^{74}$ Thus, $\mathrm{miR}-34 \mathrm{~b} / \mathrm{c}$ can inhibit MPM cells in vivo, and MPM therapy using miR-34b/c could be a future possibility.

\section{MicroRNA-205}

miR-205 appears to be involved in counteracting the EMT that MPM cells undergo when they acquire a sarcomatoid phenotype, ${ }^{75}$ a feature known to be associated with worse prognosis. ${ }^{1}$ The investigation of 58 EMPMs, 25 BMPMs, and 26 SMPMs identified underexpression of miR-205 and a switch from epithelial to mesenchymal markers in BMPM and SMPM as compared to EMPM. Furthermore, ectopic expression of miR-205 in nonmalignant mesothelial cells and MPM cell lines resulted in significant downregulation of the mesenchymal transcription factors ZEB1 and ZEB2, consequent upregulation of E-cadherin expression, and impaired migration and invasion. ${ }^{75}$ These results imply that miR-205 downregulation is an important event in the EMT of MPM cells and acquisition of a particularly aggressive behavior, which could be exploited for diagnostics, prognostics, and treatment of BMPM and SMPM.

\section{Additional miRNAs with biomarker potential in MPM}

Recently, additional studies highlighting the many-sided roles of miRNAs in MPM pathogenesis were published.

Methylation-induced silencing of the putative TSG ZIC1 leading to the overexpression of its targets miR-23a and miR-27a was recently described in MPM specimens and cell lines. ${ }^{76}$ Overexpression of miR-23a was a negative prognostic factor for patients with MPM, and reexpressing ZIC1 in MPM cells inhibited miR-23a and miR-27a expression and suppressed proliferation and invasion. ${ }^{76}$ Therefore, these two miRNAs, which have shown tumorigenic properties in other cancer types, seem to contribute to the development of MPM too. If further studies can confirm that these miRNAs act as oncomiRs in MPM, they could represent diagnostic markers and attractive therapeutic targets via miR antagonists.

As mentioned earlier, higher miR-193a-3p levels in MPM can help to differentiate this cancer from pulmonary adenocarcinoma; ${ }^{17}$ however, this miRNA was newly reported to be significantly downregulated in MPM when compared to normal pleura. ${ }^{77}$ Transfecting MPM cell lines with miR193a-3p-mimics decreased the expression of antiapoptotic $M C L-1$ gene and induced cell death, whereas targeted delivery of miR-193a-3p-containing nanocells induced apoptosis and suppressed the growth of subcutaneous MPM xenografts in nude mice. ${ }^{77}$ Thus, miR-193a-3p acts as a tumor suppressor in MPM and may potentially be useful in its diagnostics and targeted therapy, utilizing the same delivery approach currently being tested with promising preliminary results in a Phase I clinical trial for a miR-15/16-derived mimic. ${ }^{78}$

miR-223 has shown both stimulatory and inhibitory effects on inflammation and carcinogenesis in different organs. ${ }^{79,80}$ Recently, diminished levels of miR-223 in MPM 
cell lines, surgical specimens, and cells from pleural effusions were observed when compared with respective nonmalignant controls. ${ }^{81}$ The authors also showed that miR-223 can be induced by c-Jun N-terminal kinase signaling and inhibits the proliferation and motility of MPM cell lines by repressing the microtubule regulator stathmin. ${ }^{81}$ Thus, if validated, both miR-223 and stathmin could be future MPM biomarkers and, being modulated by the c-Jun N-terminal kinase signaling pathway, they could be potential therapeutic targets.

An additional study comparing the expression of miRNAs and mRNAs in 18 snap-frozen MPM specimens and six benign asbestos-induced pleural effusions found significant upregulation of let-7a, miR-125a-5p, miR-320, and miR-484 as well as the overexpression of c-Met transcript in the former group. ${ }^{82}$ Moreover, integrated analysis of miRNA-mRNA interactions revealed multiple altered targets within the Notch signaling pathway. The pathogenetic and diagnostic values of these findings await validation in larger, more homogeneous, and possibly patient-matched cohorts of MPM/NNP samples.

Recent bioinformatics and interactome network analysis of differentially expressed miRNAs in chronic pleuritis, atypical mesothelial hyperplasia, and MPM showed that these three interconnected pathological processes share downregulation of certain cancer-related miRNAs and their associated biological networks. ${ }^{83}$ Most of the downregulated miRNAs in MPM targeted the signaling activation molecule MAPK1, the transcription factor ETS1, the EMT-associated molecule FZDA, and the proinflammatory enzyme COX-2, and these were among the overlapping miRNA targets in atypical hyperplasia and chronic pleuritis. ${ }^{83}$ This network analysis supports the notion of a potential combinatory effect of deregulated miRNAs in MPM pathogenesis and indicates potential molecular mechanistic links of pleural inflammation and atypical hyperplasia with the development of MPM.

\section{Circulating miRNAs as diagnostic biomarkers of MPM}

Tumor-specific profiles of circulating miRNAs as minimally invasive diagnostic, prognostic, or predictive biomarkers have been proposed in several types of cancer. However, only few studies on circulating miRNAs in MPM have been performed. Those concerning miR-126 and miR-34 have been mentioned earlier. ${ }^{29,30,70}$

Significant underexpression of miR-103 was reported in the cell fraction of peripheral blood from patients with MPM, when compared to asbestos-exposed healthy individuals and controls from the general population. ${ }^{84}$ Evaluating the diagnostic performance, miR-103 discriminated patients with
MPM from asbestos-exposed individuals with a sensitivity of $83 \%$ and a specificity of $71 \%$ and from the general population with a sensitivity of $78 \%$ and a specificity of $76 \%{ }^{84}$

The ability of miRNAs in the cell-free fraction to serve as diagnostic biomarkers of MPM was also explored. Kirschner et al profiled plasma samples from five patients with MPM and three healthy individuals, revealing that the levels of two previously MPM-associated miRNAs (miR-29c* and -92a) and 15 novel miRNAs were increased in the former. ${ }^{56}$ Further validation by RT-qPCR in an expanded group of 45 plasma samples disclosed increased expression of the novel miR$625-3 p$ as a potential diagnostic biomarker discriminating MPM from healthy individuals with a sensitivity of $73 \%$, a specificity of $79 \%$, and an overall accuracy of $82 \% .{ }^{56}$ Notably, miR-625-3p upregulation was also detected in surgical MPM specimens, corroborating the diagnostic potential of circulating miR-625-3p.

Interestingly, miRNAs seem capable of successfully complementing soluble proteins, such as mesothelin, possessing high specificity but low sensitivity of $60 \%$ for early detection of MPM in serum or pleural exudates, thereby improving the accuracy of MPM diagnosis. ${ }^{29}$ The combination of mesothelin and the aforementioned miR-103 (now with the new miRBase ID: miR-103a-3p) as blood-based biomarkers could discriminate EMPM and BMPM from asbestos-exposed controls with improved specificity and sensitivity as compared to either individual marker alone. ${ }^{85}$ Moreover, the combined triple measurement of SMRPs with methylated thrombomodulin promoter and miR-126 in the serum was reported to significantly enhance the performance of serum SMRPs in distinguishing patients with MPM from asbestos-exposed subjects and healthy controls. ${ }^{86}$

Although the possibility of measuring serum levels of miRNAs for diagnostic purposes needs to be analyzed prospectively, these results support the notion that circulating miRNAs may represent promising and less "invasive" biomarkers in MPM.

\section{Prognostic impact of miRNAs in MPM}

As noticed earlier, miRNA expression profiles in MPM could also aid in predicting the patient outcome. ${ }^{15,20,29,59,63}$ Recently, miRNA profiling in tissue specimens from patients with MPM with short- and long-term postoperative survival was combined with binary logistic regression to identify a 6-miR signature (miR-21-5p, -23a-3p, -30e-5p, -221-3p, -222-3p, and $-31-5 p$ ) called miR-score, which was capable of predicting a survival of $\geq 20$ months with an accuracy of $92.3 \%$ for 
extrapleural pneumonectomy and $71.9 \%$ for pleurectomy/ decortication. ${ }^{87}$ When the miR-score was associated with clinical prognostic parameters (histology, age, and sex), the predictive accuracy in a validation set was improved from $76.3 \%$ to $87.3 \%$. Therefore, the authors proposed the miRscore as novel accurate prognostic biomarker in MPM. ${ }^{87}$

\section{Current limitations and possible new applications of miRNAs in MPM diagnostics}

As mentioned earlier, one limitation of the reported studies is that they have used different methodological platforms of miRNA microarrays and/or RT-qPCR and types of specimens, thereby generating different miR signatures in MPM cells that are difficult to compare. A common problem to studies on MPM tissue specimens is the difficult acquisition of patientmatched NNP samples due to the diffused growth of MPM. Moreover, MPM is a heterogeneous and poorly circumscribed tumor tissue containing a mixture of malignant cells, inflammatory cells, and reactive stromal structures. Thus, RT-qPCRbased analyses (miRNA arrays or TaqMan ${ }^{\circledR}$ assays) of RNA extracts may provide results that are not always reliable for discriminating miRNA changes occurring within MPM cells from those associated with nonneoplastic components. ${ }^{88}$ To compensate for these confounding factors, the use of tissue samples enriched with cancer cells (representing at least $50 \%$ of cells in the tissue) is recommendable, though it does not eliminate the risk of drawing deceptive conclusions. Tumor content enrichment by laser capture microdissection followed by accurate quality verification of intact miRNA fraction has also been proposed. ${ }^{87}$

A possible solution to avoid these drawbacks and enhance the feasibility and accuracy of routinely testing miRNAs in FFPE MPM specimens is to investigate them directly on tissue sections by ISH with specific locked nucleic acidmodified probes. ${ }^{89}$ ISH provides information regarding contextual tissue distribution and cellular origin of candidate $\mathrm{miR}$ biomarkers and better interpretation of their functions and interactions in complex multicellular tissue samples. Furthermore, the codetection of miRNA targets by IHC can be employed to further improve the knowledge of the biological function of miRNAs and colocalization within the tumor. ${ }^{88,90}$ A diagnostic assay combining ISH of deregulated miRNAs and IHC-based detection of related targets in tissue biopsies could further improve the differential diagnosis of RMP vs MPM, by also enabling the analysis of samples with low tumor cell content and by helping to monitor the changes of potential miRNA targets in a therapeutic setting. ${ }^{90}$
However, to construct a combined ISH-IHC assay, the related targets of candidate miR biomarkers need to be identified in MPM and their diagnostic potential assessed. In this respect, the number of miRNA targets in MPM that have been validated by in vitro experiments is currently small. To date, direct physical miRNA-mRNA interaction has only been evidenced in MPM for miR-126-IRS1 and miR-145-OCT4 (Table 2). Some evidence has been provided for $L A T 1$ as target of miR-126, BCL2 and CCND1 of miR-16, PPP6C of miR-31, MCL1 of miR-193-3p, STMN1 of miR-223, and for few additional targets of other miRNAs. ${ }^{16,20,34,45,57,60,62,77,81}$ The detection of biologically active miRNA targets is challenging and initially involves the use of computational target prediction, although some algorithms may not be accurate enough and require caution in interpreting the obtained results. Combining the predictions of several algorithms may improve the accuracy, yet this approach does not consider possible cell type-specific obstruction of the target site by RNA-binding proteins. Given that miRNAs primarily act to repress target mRNAs, one method to achieve better prediction accuracy is to compare changes in gene expression and miRNA levels of patient-matched MPM/NNP specimens.

General pitfalls concerning circulating miRNAs as biomarkers, not least the need of more validation and the contribution to miRNA secretion by blood cells, together with possible solutions, have been extensively reviewed elsewhere. ${ }^{91}$

\section{Conclusion}

In summary, despite several aspects of the involvement of miRNAs in mesothelioma genesis remain contentious, the aforementioned results illustrate that miRNAs have a great potential as diagnostic and prognostic biomarkers in MPM. Because of the inherent weaknesses of RT-qPCR-based detection methods, further studies assessing the expression and distribution of miRNAs by ISH and codetection of their respective targets by IHC, as well as validation of miRNA targets in vitro are necessary to fully reveal the potential clinical utility of miRNAs and their related targets. Moreover, circulating miRNAs are attractive, though not yet optimized, "noninvasive" biomarkers for diagnostics and monitoring of patients with MPM.

\section{Disclosure}

The authors report no conflicts of interest in this work.

\section{References}

1. Travis WD, Brambilla E, Burke AP, Marx A, Nicholson AG, editors. WHO Classification of Tumours of the Lung, Pleura, Thymus and Heart. 4th ed. Lyon: IARC Press; 2015. 
2. Scherpereel A, Astoul P, Baas P, et al; European Respiratory Society/ European Society of Thoracic Surgeons Task Force. European Respiratory Society/European Society of Thoracic Surgeons Task F: Guidelines of the European Respiratory Society and the European Society of Thoracic Surgeons for the management of malignant pleural mesothelioma. Eur Respir J. 2010;35(3):479-495.

3. Zimling ZG, Sørensen JB, Gerds TA, Bech C, Andersen CB, SantoniRugiu E. A biomarker profile for predicting efficacy of cisplatinvinorelbine therapy in malignant pleural mesothelioma. Cancer Chemother Pharmacol. 2012;70(5):743-754.

4. Remon J, Reguart N, Corral J, Lianes P. Malignant pleural mesothelioma: new hope in the horizon with novel therapeutic strategies. Cancer Treat Rev. 2015;41(1):27-34.

5. Zimling ZG, Jørgensen A, Santoni-Rugiu E. The diagnostic value of immunohistochemically detected methylthioadenosine phosphorylase deficiency in malignant pleural mesotheliomas. Histopathology. 2012;60(6B):E96-E105.

6. Churg A, Sheffield BS, Galateau-Salle F. New markers for separating benign from malignant mesothelial proliferations: are we there yet? Arch Pathol Lab Med. Epub 2015 Aug 19.

7. Lu J, Getz G, Miska EA, et al. MicroRNA expression profiles classify human cancers. Nature. 2005;435(7043):834-838.

8. Yu SL, Chen HY, Chang GC, et al. MicroRNA signature predicts survival and relapse in lung cancer. Cancer Cell. 2008;13(1): 48-57.

9. Bartel DP. MicroRNAs: target recognition and regulatory functions. Cell. 2009;136(2):215-233.

10. Griffiths-Jones S, Grocock RJ, van Dongen S, Bateman A, Enright AJ. miRBase: microRNA sequences, targets and gene nomenclature Nucleic Acids Res. 2006;34(Database issue):D140-D144.

11. Jansson MD, Lund AH. MicroRNA and cancer. Mol Oncol. 2012;6(6): 590-610.

12. Siebolts U, Varnholt H, Drebber U, Dienes HP, Wickenhauser C, Odenthal $\mathrm{M}$. Tissues from routine pathology archives are suitable for microRNA analyses by quantitative PCR. J Clin Pathol. 2009;62(1): 84-88.

13. Schwarzenbach H, Nishida N, Calin GA, Pantel K. Clinical relevance of circulating cell-free microRNAs in cancer. Nat Rev Clin Oncol. 2014;11(3):145-156.

14. Guled M, Lahti L, Lindholm PM, et al. CDKN2A, NF2, and JUN are dysregulated among other genes by miRNAs in malignant mesothelioma-A miRNA microarray analysis. Genes Chromosomes Cancer. 2009;48(7):615-623.

15. Busacca S, Germano S, De Cecco L, et al. MicroRNA signature of malignant mesothelioma with potential diagnostic and prognostic implications. Am J Respir Cell Mol Biol. 2010;42(3):312-319.

16. Balatti V, Maniero S, Ferracin M, et al. MicroRNAs dysregulation in human malignant pleural mesothelioma. J Thorac Oncol. 2011;6(5) 844-851.

17. Benjamin H, Lebanony D, Rosenwald S, et al. A diagnostic assay based on microRNA expression accurately identifies malignant pleural mesothelioma. J Mol Diagn. 2010;12(6):771-779.

18. Gee GV, Koestler DC, Christensen BC, et al. Downregulated microRNAs in the differential diagnosis of malignant pleural mesothelioma. Int J Cancer. 2010;127(12):2859-2869.

19. Andersen M, Grauslund M, Muhammad-Ali M, et al. Are differentially expressed microRNAs useful in the diagnostics of malignant pleural mesothelioma? APMIS. 2012;120(9):767-769.

20. Andersen M, Grauslund M, Ravn J, Sørensen JB, Andersen CB, SantoniRugiu E. Diagnostic potential of miR-126, miR-143, miR-145, and miR-652 in malignant pleural mesothelioma. J Mol Diagn. 2014;16(4):418-430.

21. Miko E, Margitai Z, Czimmerer Z, et al. miR-126 inhibits proliferation of small cell lung cancer cells by targeting SLC7A5. FEBS Lett. 2011;585(8):1191-1196.

22. Kaira K, Oriuchi N, Takahashi T, et al. L-Type amino acid transporter 1 (LAT1) expression in malignant pleural mesothelioma. Anticancer Res. 2011;31(12):4075-4082.
23. Li XM, Wang AM, Zhang J, Yi H. Down-regulation of miR-126 expression in colorectal cancer and its clinical significance. Med Oncol. 2011;28(4):1054-1057.

24. Tavazoie SF, Alarcón C, Oskarsson T, et al. Endogenous human microRNAs that suppress breast cancer metastasis. Nature. 2008;451(7175): $147-152$.

25. Feng R, Chen X, Yu Y, et al. miR-126 functions as a tumour suppressor in human gastric cancer. Cancer Lett. 2010;298(1):50-63.

26. Parker LH, Schmidt M, Jin SW, et al. The endothelial-cell-derived secreted factor $E g f l 7$ regulates vascular tube formation. Nature. 2004;428(6984):754-758.

27. Saito Y, Friedman JM, Chihara Y, Egger G, Chuang JC, Liang G. Epigenetic therapy upregulates the tumor suppressor microRNA-126 and its host gene EGFL7 in human cancer cells. Biochem Biophys Res Commun. 2009;379(3):726-731.

28. Andersen M, Trapani D, Ravn J, et al. Methylation-associated silencing of microRNA-126 and its host gene EGFL7 in malignant pleural mesothelioma. Anticancer Res. 2015;35(11):6223-6229.

29. Santarelli L, Strafella E, Staffolani S, et al. Association of MiR-126 with soluble mesothelin-related peptides, a marker for malignant mesothelioma. PLoS One. 2011;6(4):e18232.

30. Tomasetti M, Staffolani S, Nocchi L, et al. Clinical significance of circulating miR-126 quantification in malignant mesothelioma patients. Clin Biochem. 2012;45(7-8):575-581.

31. Sun Y, Bai Y, Zhang F, Wang Y, Guo Y, Guo L. miR-126 inhibits nonsmall cell lung cancer cells proliferation by targeting EGFL 7. Biochem Biophys Res Commun. 2010;391(3):1483-1489.

32. Zhu N, Zhang D, Xie H, et al. Endothelial-specific intron-derived miR126 is down-regulated in human breast cancer and targets both VEGFA and PIK3R2. Mol Cell Biochem. 2011;351(1-2):157-164.

33. Jiao LR, Frampton AE, Jacob J, et al. MicroRNAs targeting oncogenes are down-regulated in pancreatic malignant transformation from benign tumors. PLoS One. 2012;7(2):e32068.

34. Tomasetti M, Nocchi L, Staffolani S, et al. MicroRNA-126 suppresses mesothelioma malignancy by targeting IRS1 and interfering with mitochondrial function. Antioxid Redox Signal. 2014;21(15):2109-2125.

35. Bao B, Azmi AS, Ali S, et al. The biological kinship of hypoxia with CSC and EMT and their relationship with deregulated expression of miRNAs and tumor aggressiveness. Biochim Biophys Acta. 2012; 1826(2):272-296

36. LiT, Li H, Wang Y, et al. The expression of CXCR4, CXCL12 and CXCR7 in malignant pleural mesothelioma. $J$ Pathol. 2011;223(4):519-530.

37. Li Z, Li N, Wu M, Li X, Luo Z, Wang X. Expression of miR-126 suppresses migration and invasion of colon cancer cells by targeting CXCR4. Mol Cell Biochem. 2013;381(1-2):233-242.

38. Zhang Y, Yang P, Sun T, et al. miR-126 and miR-126* repress recruitment of mesenchymal stem cells and inflammatory monocytes to inhibit breast cancer metastasis. Nat Cell Biol. 2013;15(3):284-294.

39. Guo YH, Zhang C, Shi J, et al. Abnormal activation of the EGFR signaling pathway mediates the downregulation of miR145 through the ERK1/2 in non-small cell lung cancer. Oncol Rep. 2014;31(4):1940-1946.

40. Kojima S, Enokida H, Yoshino H, et al. The tumor-suppressive microRNA-143/145 cluster inhibits cell migration and invasion by targeting GOLM1 in prostate cancer. J Hum Genet. 2014;59(2):78-87.

41. Lu R, Ji Z, Li X, et al. miR-145 functions as tumor suppressor and targets two oncogenes, $A N G P T 2$ and NEDD9, in renal cell carcinoma. J Cancer Res Clin Oncol. 2014;140(3):387-397.

42. Zhang J, Sun Q, Zhang Z, Ge S, Han ZG, Chen WT. Loss of microRNA$143 / 145$ disturbs cellular growth and apoptosis of human epithelial cancers by impairing the MDM2-p53 feedback loop. Oncogene. 2013; 32(1):61-69.

43. Kent OA, Chivukula RR, Mullendore M, et al. Repression of the miR-143/145 cluster by oncogenic Ras initiates a tumor-promoting feed-forward pathway. Genes Dev. 2010;24(24):2754-2759.

44. Chen Z, Zeng H, Guo Y, et al. miRNA-145 inhibits non-small cell lung cancer cell proliferation by targeting c-Myc. J Exp Clin Cancer Res. 2010;29:151. 
45. Cioce M, Ganci F, Canu V, et al. Protumorigenic effects of mir-145 loss in malignant pleural mesothelioma. Oncogene. 2014;33(46): 5319-5331.

46. Casarsa C, Bassani N, Ambrogi F, et al. Epithelial-to-mesenchymal transition, cell polarity and stemness-associated features in malignant pleural mesothelioma. Cancer Lett. 2011;302(2):136-143.

47. Lulla RR, Costa FF, Bischof JM, et al. Identification of differentially expressed microRNAs in osteosarcoma. Sarcoma. 2011;2011: 732690 .

48. Gaedcke J, Grade M, Camps J, et al. The rectal cancer microRNAomemicroRNA expression in rectal cancer and matched normal mucosa. Clin Cancer Res. 2012;18(18):4919-4930.

49. McDermott AM, Miller N, Wall D. Identification and validation of oncologic miRNA biomarkers for luminal A-like breast cancer. PLoS One. 2014;9(1):e87032.

50. Kleivi Sahlberg K, Bottai G, Naume B, et al. A serum microRNA signature predicts tumor relapse and survival in triple-negative breast cancer patients. Clin Cancer Res. 2015;21(5):1207-1214.

51. Bernardo BC, Nguyen SS, Winbanks CE, et al. Therapeutic silencing of miR-652 restores heart function and attenuates adverse remodeling in a setting of established pathological hypertrophy. FASEB J. 2014;28(12): 5097-5110

52. Kettunen E, Nissén AM, Ollikainen T, et al. Gene expression profiling of malignant mesothelioma cell lines: cDNA array study. Int J Cancer. 2001;91(4):492-496.

53. Xiong L, Edwards CK, Zhou L. The biological function and clinical utilization of CD147 in human diseases: a review of the current scientific literature. Int J Mol Sci. 2014;15(10):17411-17441.

54. Pinheiro C, Longatto-Filho A, Soares TR, et al. CD147 immunohistochemistry discriminates between reactive mesothelial cells and malignant mesothelioma. Diagn Cytopathol. 2012;40(6):478-483.

55. XuY, Zheng M, Merritt RE, et al. miR-1 induces growth arrest and apoptosis in malignant mesothelioma. Chest. 2013;144(5):1632-1643.

56. Kirschner MB, Cheng YY, Badrian B, et al. Increased circulating miR-625-3p: a potential biomarker for patients with malignant pleural mesothelioma. J Thorac Oncol. 2012;7(7):1184-1191.

57. Reid G, Pel ME, Kirschner MB, et al. Restoring expression of miR-16: a novel approach to therapy for malignant pleural mesothelioma. Ann Oncol. 2013;24(12):3128-3135.

58. Cimmino A, Calin GA, Fabbri M, et al. miR-15 and miR-16 induce apoptosis by targeting BCL2. Proc Natl Acad Sci USA. 2005;102(39): 13944-13949.

59. Liu Q, Fu H, Sun F, et al. miR-16 family induces cell cycle arrest by regulating multiple cell cycle genes. Nucleic Acids Res. 2008;36(16): 5391-5404.

60. Pass HI, Goparaju C, Ivanov S, et al. hsa-miR-29c* is linked to the prognosis of malignant pleural mesothelioma. Cancer Res. 2010;70(5): 1916-1924.

61. Fabbri M, Garzon R, Cimmino A, et al. MicroRNA-29 family reverts aberrant methylation in lung cancer by targeting DNA methyltransferases 3A and 3B. Proc Natl Acad Sci U S A. 2007;104(40):15805-15810.

62. Ivanov SV, Goparaju CM, Lopez P, et al. Pro-tumorigenic effects of miR-31 loss in mesothelioma. J Biol Chem. 2010;285(30):22809-22817.

63. MacKeigan JP, Murphy LO, Blenis J. Sensitized RNAi screen of human kinases and phosphatases identifies new regulators of apoptosis and chemoresistance. Nat Cell Biol. 2005;7(6):591-600.

64. Matsumoto S, Nabeshima K, Hamasaki M, Shibuta T, Umemura T. Upregulation of microRNA-31 associates with a poor prognosis of malignant pleural mesothelioma with sarcomatoid component. Med Oncol. 2014;31(12):303.

65. He L, He X, Lim LP, et al. A microRNA component of the p53 tumour suppressor network. Nature. 2007;447(7148):1130-1134.

66. Christoffersen NR, Shalgi R, Frankel LB, et al. p53-independent upregulation of miR-34a during oncogene-induced senescence represses MYC. Cell Death Differ. 2010;17(2):236-245.

67. Wang R, Ma J, Wu Q, et al. Functional role of miR-34 family in human cancer. Curr Drug Targets. 2013;14(10):1185-1191.
68. Okada N, Lin CP, Ribeiro MC, et al. A positive feedback between p53 and miR-34 miRNAs mediates tumor suppression. Genes Dev. 2014;28(5):438-450.

69. Kubo T, Toyooka S, Tsukuda K, et al. Epigenetic silencing of microRNA$34 \mathrm{~b} / \mathrm{c}$ plays an important role in the pathogenesis of malignant pleural mesothelioma. Clin Cancer Res. 2011;17(15):4965-4974.

70. Muraoka T, Soh J, Toyooka S, et al. The degree of microRNA-34b/c methylation in serum-circulating DNA is associated with malignant pleural mesothelioma. Lung Cancer. 2013;82(3):485-490.

71. Maki Y, Asano H, Toyooka S, et al. MicroRNA miR-34b/c enhances cellular radiosensitivity of malignant pleural mesothelioma cells. Anticancer Res. 2012;32(11):4871-4875.

72. Tanaka N, Toyooka S, Soh J, et al. Downregulation of microRNA-34 induces cell proliferation and invasion of human mesothelial cells. Oncol Rep. 2013;29(6):2169-2174.

73. Menges CW, Kadariya Y, Altomare D, et al. Tumor suppressor alterations cooperate to drive aggressive mesotheliomas with enriched cancer stem cells via a p53-miR-34a-c-Met axis. Cancer Res. 2014;74(4): 1261-1271.

74. Ueno T, Toyooka S, Fukazawa T, et al. Preclinical evaluation of microRNA-34b/c delivery for malignant pleural mesothelioma. Acta Med Okayama. 2014;68(1):23-26.

75. Fassina A, Cappellesso R, Guzzardo V, et al. Epithelial-mesenchymal transition in malignant mesothelioma. Mod Pathol. 2012;25(1): 86-99.

76. Cheng YY, Kirschner MB, Cheng NC, et al. ZIC1 is silenced and has tumor suppressor function in malignant pleural mesothelioma. JThorac Oncol. 2013;8(10):1317-1328.

77. Williams M, Kirschner MB, Cheng YY, et al. miR-193a-3p is a potential tumor suppressor in malignant pleural mesothelioma. Oncotarget. 2015;6(27):23480-23495.

78. Kao SC, Fulham M, Wong K, et al. A significant metabolic and radiological response after a novel targeted microrna-based treatment approach in malignant pleural mesothelioma. Am J Respir Crit Care Med. 2015;191(12):1467-1469.

79. Haneklaus M, Gerlic M, O’Neill LA, Masters SL. miR-223: infection, inflammation and cancer. J Intern Med. 2013;274(3):215-226.

80. Taïbi F, Metzinger-Le Meuth V, Massy ZA, Metzinger L. miR-223: an inflammatory oncomiR enters the cardiovascular field. Biochim Biophys Acta. 2014;1842(7):1001-1009

81. Birnie KA, Yip YY, Ng DC, et al. Loss of mir-223 and JNK signalling contribute to elevated stathmin in malignant pleural mesothelioma. Mol Cancer Res. 2015;13(7):1106-1118.

82. Ak G, Tomaszek SC, Kosari F, et al. MicroRNA and mRNA features of malignant pleural mesothelioma and benign asbestos-related pleural effusion. Biomed Res Int. 2015;2015:635748.

83. Ramírez-Salazar EG, Salinas-Silva LC, Vázquez-Manríquez ME, et al. Analysis of microRNA expression signatures in malignant pleural mesothelioma, pleural inflammation, and atypical mesothelial hyperplasia reveals common predictive tumorigenesis-related targets. Exp Mol Pathol. 2014;97(3):375-385.

84. Weber DG, Johnen G, Bryk O, Jöckel KH, Brüning T. Identification of miRNA-103 in the cellular fraction of human peripheral blood as a potential biomarker for malignant mesothelioma - a pilot study. PLoS One. 2012;7(1):e30221.

85. Weber DG, Casjens S, Johnen G, et al. Combination of MiR-103a-3p and mesothelin improves the biomarker performance of malignant mesothelioma diagnosis. PLoS One. 2014;9(12):e114483s.

86. Santarelli L, Staffolani S, Strafella E, et al. Combined circulating epigenetic markers to improve mesothelin performance in the diagnosis of malignant mesothelioma. Lung Cancer. Epub 2015 Sep 25.

87. Kirschner MB, Cheng YY, Armstrong NJ, et al. MiR-score: a novel 6-microRNA signature that predicts survival outcomes in patients with malignant pleural mesothelioma. Mol Oncol. 2015;9(3):715-726.

88. Sempere LF, Preis M, Yezefski T, et al. Fluorescence-based codetection with protein markers reveals distinct cellular compartments for altered MicroRNA expression in solid tumors. Clin Cancer Res. 2010;16(16): 4246-4255. 
89. Nielsen BS. MicroRNA in situ hybridization. Methods Mol Biol. 2012;822:67-84.

90. Nielsen BS, Holsmtrøm K. Combined microRNA in situ hybridization and immunohistochemical detection of protein markers. Methods $\mathrm{Mol}$ Biol. 2013;986:353-365.
91. Witwer KW. Circulating microRNA biomarker studies: pitfalls and potential solutions. Clin Chem. 2015;61(1):56-63.

Current Biomarker Findings

\section{Publish your work in this journal}

Current Biomarker Findings is an international, peer-reviewed, open access journal publishing original research, reports, reviews and commentaries on all areas of biomarker research. The manuscript management system is completely online and includes a very quick and fair

\section{Dovepress}

peer-review system. Visit http://www.dovepress.com/testimonials.php to read real quotes from published authors. 